\title{
Generalized Weyl solutions
}

\author{
Roberto Emparan* \\ Theory Division, CERN, CH-1211 Geneva 23, Switzerland
}

Harvey S. Reall

Physics Department, Queen Mary College, Mile End Road, London E1 4NS, United Kingdom

(Received 8 November 2001; published 3 April 2002)

\begin{abstract}
It was shown by Weyl that the general static axisymmetric solution of the vacuum Einstein equations in four dimensions is given in terms of a single axisymmetric solution of the Laplace equation in three-dimensional flat space. Weyl's construction is generalized here to arbitrary dimension $D \geqslant 4$. The general solution of the $D$-dimensional vacuum Einstein equations that admits $D-2$ orthogonal commuting non-null Killing vector fields is given either in terms of $D-3$ independent axisymmetric solutions of Laplace's equation in threedimensional flat space or by $D-4$ independent solutions of Laplace's equation in two-dimensional flat space. Explicit examples of new solutions are given. These include a five-dimensional asymptotically flat "black ring" with an event horizon of topology $S^{1} \times S^{2}$ held in equilibrium by a conical singularity in the form of a disk.

DOI: $10.1103 /$ PhysRevD.65.084025

PACS number(s): 04.50.+h, 04.20.Jb, 04.70.Bw
\end{abstract}

\section{INTRODUCTION}

Exact solutions play an important role in general relativity. Examining properties of exact solutions has led to deep insights into the nature of spacetime that would have been hard to arrive at by other means. For example, much of the progress made in understanding properties of black holes in the 1960s and 1970s relied on the existence of the KerrNewman solution. The standard model of cosmology is built on Friedmann-Robertson-Walker solutions. Examining properties of Bianchi cosmologies has led to insight into how inflation dissipates anisotropy.

Much effort has been devoted to developing techniques for finding exact solutions in four dimensions [1,2]. One of the earliest results in this direction was obtained by Weyl [3], who found the general static axisymmetric solution of the vacuum Einstein equations:

$$
d s^{2}=-e^{2 U} d t^{2}+e^{-2 U}\left(e^{2 \gamma}\left(d r^{2}+d z^{2}\right)+r^{2} d \phi^{2}\right),
$$

where $U(r, z)$ is an arbitrary axisymmetric solution of Laplace's equation in a three-dimensional flat space with metric

$$
d s^{2}=d r^{2}+r^{2} d \phi^{2}+d z^{2},
$$

and $\gamma$ satisfies

$$
\begin{aligned}
& \frac{\partial \gamma}{\partial r}=r\left[\left(\frac{\partial U}{\partial r}\right)^{2}-\left(\frac{\partial U}{\partial z}\right)^{2}\right], \\
& \frac{\partial \gamma}{\partial z}=2 r \frac{\partial U}{\partial r} \frac{\partial U}{\partial z} .
\end{aligned}
$$

\footnotetext{
*Also at Departamento de Física Teórica, Universidad del País Vasco, E-48080, Bilbao, Spain.
}

The solution of these equations is given by a line integral. Since $U$ is harmonic, it can be regarded as a Newtonian potential produced by certain (axisymmetric) sources. For example, the Schwarzschild solution corresponds to taking the source for $U$ to be a thin rod on the $z$ axis with mass $1 / 2$ per unit length.

Nowadays, interest in solutions of general relativity is no longer restricted to four dimensions. Many interesting solutions of higher dimensional supergravity theories have been found. In spite of this, there are basic questions concerning the nature of gravity in higher dimensions that remain unanswered. In four dimensions, it can be proved that each connected component of the event horizon of an asymptotically flat spacetime satisfying the dominant energy condition has topology $S^{2}$ [4]. The proof relies on the Gauss-Bonnet theorem applied to a constant time slice through the horizon, and is therefore invalid in higher dimensions. A different approach to rule out nonspherical topologies is based on the notion of "topological censorship" [5]. However, this argument is typically phrased in terms of noncontractible loops that begin and end at infinity, and which would thread through a toroidal horizon. In higher dimensions, one can always unlink two loops by moving them apart in a fourth spatial direction. This suggests that it might be possible for the event horizon to have nonspherical topology in higher dimensions. Indeed, in [6], it was argued that the horizon of a time-symmetric black hole in five dimensions must have topology given by a connected sum of $S^{3}$ and $S^{1} \times S^{2}$ terms, subject to the weak energy condition. Nevertheless, no example of an asymptotically flat solution with a nonspherical event horizon has ever been found. ${ }^{1}$ One aim of the present

\footnotetext{
${ }^{1}$ A solution with a regular, though degenerate, horizon of topology $S^{1} \times S^{2}$ has been found in [7]. Although not asymptotically flat, this solution has a spacelike infinity of topology $S^{3}$, which distinguishes it from examples in which horizons of nonspherical topology are constructed by taking spacelike infinity to have nonspherical topology.
} 
paper is to provide an example of such a spacetime.

We will, for simplicity, consider only the vacuum Einstein equations. A lot of work has been devoted to finding exact solutions of these equations in dimensions $D>4$. Most of this work has looked for solutions with a Kaluza-Klein (KK) interpretation, i.e., solutions with a Killing vector field along which one can perform dimensional reduction to get a sensible lower dimensional spacetime. For example, KK black hole solutions were discussed in [8] and a KK monopole solution was presented in [9]. KK generalizations of the $C$ metric [10] and Ernst metric [11] were presented in [12]. Other axially symmetric solutions in KK theory have been discussed in [13-16].

Less work has been devoted to finding solutions of the $D$-dimensional vacuum Einstein equations that do not admit a KK interpretation, either because they do not admit an appropriate Killing vector field along which KK reduction can be performed, or because the reduced spacetime has pathological features. Examples of such spacetimes are provided by higher dimensional versions of the Schwarzschild and Kerr black holes $[17,18]$. When Wick rotated, these solutions do admit KK interpretations as describing instabilities of the KK vacuum [19] or of KK magnetic fields [20,21], but their most natural interpretation is certainly as higher dimensional black holes.

The purpose of the present paper is to obtain and analyze the higher dimensional analogues of Weyl's class of solutions. Depending on which feature of Weyl's class one focuses on, there are several directions in which one can try to extend it to higher dimensions. One possibility is to seek the class of $D$-dimensional solutions that are static and axisymmetric, in the sense that they admit an isometry group $\mathbf{R}$ $\times O(D-2)$ (with $\mathbf{R}$ being time translations). However, this has been tried before [22] without success. Instead, observe that Weyl's solutions can be characterized as having two orthogonal commuting Killing vector fields. Hence an alternative way to generalize Weyl's solutions to higher dimensions is to find all solutions of the vacuum Einstein equations that admit $D-2$ orthogonal commuting Killing vector fields. This is done in Sec. II of this paper.

As in four dimensions, the higher-dimensional Weyl class of solutions is parametrized in terms of axisymmetric harmonic functions in an auxiliary flat space. Actually, there are two classes of Weyl solutions in higher dimensions. The first, and the most interesting one, is parametrized in terms of $D$ -3 harmonic functions in three-dimensional flat space, and is the natural analogue of the $D=4$ Weyl solutions discussed above. The second class of solutions (discussed in Appendix B) is parametrized in terms of $D-4$ harmonic functions in two-dimensional flat space, and therefore has no $D=4$ analogue.

Although Weyl's construction in $D=4$ describes an infinite class of solutions, most of them are unphysical in the sense that they are not asymptotically flat, or have naked curvature singularities on the axis of symmetry. ${ }^{2}$ The same is

\footnotetext{
${ }^{2}$ E.g., a spherically symmetric point source for $U$ results in a singular, nonspherical Chazy-Curzon particle.
}

true for $D>4$. In order to select candidate Weyl solutions that might be of physical importance, recall that for $D=4$, the harmonic function $U$ can be regarded as a Newtonian potential produced by an axisymmetric source. It turns out that the most interesting $D=4$ Weyl solutions all have sources of the same form, namely thin rods on the axis of symmetry. In Sec. III, known $D>4$ Weyl solutions of physical importance are analyzed. Their harmonic functions also always correspond to thin rods on the axis of symmetry in the auxiliary three-dimensional flat space.

A natural classification scheme for such solutions is presented in Sec. IV. In this scheme, the "zeroth" class consists simply of flat space. The first nontrivial class contains just the $D=4$ and $D=5$ Schwarzschild solutions (the $D>5$ Schwarzschild solutions do not admit $D-2$ commuting Killing vector fields and are therefore not Weyl solutions), and their Wick rotations. These Wick rotations describe objects known as "KK bubbles." If one considers the Euclideanized $D=4$ Schwarzschild solution, then the solution looks asymptotically like $\mathbf{R}^{3} \times S^{1}$, the $S^{1}$ corresponding to Euclidean time, which is periodically identified and can be regarded as a KK compactified dimension. However, the actual topology of the solution is $\mathbf{R}^{2} \times S^{2}$. The size of the two-spheres at constant radius decreases from infinity to a minimum nonzero value at the location of the Euclidean horizon, where a noncontractible $S^{2}$ lies. At this point, the KK circles smoothly round off and space cannot be continued past this radius. By adding a flat Lorentzian time direction one obtains a solution to $D=5 \mathrm{KK}$ theory where the non-contractible sphere is a static "bubble of nothing." It is known to be unstable [23]. A related solution is obtained by Wick rotating both the time and one of the ignorable angular coordinates of the five-dimensional Schwarzschild solution. The Wickrotated angle then becomes a boost coordinate and the solution describes a bubble exponentially expanding in the fivedimensional KK vacuum [19]. Its fully Euclideanized version is an instanton mediating the decay of the $\mathrm{KK}$ vacuum $M^{1,3} \times S^{1}$.

The second class of Weyl solutions contains the $D$ $=4 C$ metric as well as three new solutions. The most interesting of these is a Wick rotated version of a $D=5$ metric discussed in [24], and can also be related to the KK $C$ metric of [12]. It is a static, asymptotically flat solution with an event horizon of topology $S^{1} \times S^{2}$, i.e., it is a black ring. This is the first example of an asymptotically flat solution of the vacuum Einstein equations that has an event horizon of nonspherical topology. The solution is not entirely satisfactory since it has a conical singularity, but it will be shown in a separate publication that this singularity can be eliminated if the ring rotates [25].

The two other new solutions in the same class as the black ring and the $C$ metric both describe superpositions of black objects with static KK bubbles. These solutions are entirely regular outside an event horizon. The first is a $D=5$ solution describing a black hole sitting in the throat of a static KK bubble. The second is a $D=6$ solution describing a loop of black string with horizon topology $S^{3} \times S^{1}$ sitting in the throat of a static KK bubble. These solutions asymptote to, respectively, the KK vacua $M^{1,3} \times S^{1}$ and $M^{1,4} \times S^{1}$. Both are 
expected to be unstable. In fact, the evolution of the instability of the former solution can be obtained by a Wick rotation of the black ring. If these solutions are Euclideanized then they give new nonsingular instantons for the decay of the $T^{2}$ compactified KK vacuum in $D=5$ and $D=6$.

Many of the solutions we describe are naturally interpreted in terms of KK compactification along the orbits of one or several of the Killing vector fields. When there is more than one Killing vector field with closed orbits, one can often dimensionally reduce along different linear combinations of them. Physically distinct reduced spacetimes can therefore arise from the same higher dimensional spacetime. A good example is the KK $C$ metric and the KK Ernst solution, which are locally isometric in five dimensions [20]. With this in mind, different KK reductions of the new solutions found in this paper are briefly discussed in Sec. IV H.

Multi-black hole configurations can be readily constructed within Weyl's class, and are briefly discussed in Sec. IV I. Finally, Sec. V contains the conclusions of this work.

\section{GENERALIZED WEYL SOLUTIONS}

\section{A. Integrable submanifolds}

The first step in generalizing Weyl's construction to more than four dimensions is to find a convenient coordinate chart for the general $D$-dimensional line element admitting $D-2$ commuting Killing vector fields (orthogonality of these vector fields will not be assumed yet). This is a simple generalization of what is done in four dimensions (see [2] for a review). It will be assumed that the metric is Riemannian or Lorentzian. Let $\xi_{(i)}$ denote the Killing vector fields, $1 \leqslant i$ $\leqslant D-2$. Since these commute, it is possible to choose coordinates $\left(x^{i}, y^{1}, y^{2}\right)$ such that $\xi_{(i)}=\partial / \partial x^{i}$ with the metric coefficients depending only on $y^{1}$ and $y^{2}$.

The next step is to show that one can choose the coordinates $y^{1}$ and $y^{2}$ to span two-dimensional surfaces orthogonal to all of the $\xi_{(i)}$. In order to do this, one has to show that the two-dimensional subspaces of the tangent space orthogonal to all of the vectors $\xi_{(i)}$ are integrable, i.e., tangent to twodimensional surfaces. Sufficient conditions for integrability are supplied by the following theorem:

Theorem. Let $\xi_{(i)}, \quad 1 \leqslant i \leqslant D-2$ be commuting Killing vector fields such that for each $i$, (a) $\xi_{(1)}^{\left[\mu_{1}\right.} \xi_{(2)}^{\mu_{2}} \ldots \xi_{(D-2)}^{\mu_{D-2}} \nabla^{\nu} \xi_{(i)}^{\rho]}$ vanishes at at least one point of the spacetime (not necessarily the same point for every $i$ ), and (b) $\xi_{(i)}^{\nu} R_{\nu}^{[\rho} \xi_{(1)}^{\mu_{1}} \xi_{(2)}^{\mu_{2}} \ldots \xi_{(D-2)}^{\left.\mu_{D-2}\right]}=0$. Then the two planes orthogonal to the $\xi_{(i)}$ are integrable.

The proof of this theorem is a straightforward generalization of the corresponding theorem in four dimensions, as given in [2]. In this paper, only vacuum solutions of the Einstein equations will be considered so condition (b) is trivially satisfied. Condition (a) is less obvious; in four dimensions it is usually assumed that one of the Killing vector fields is an angular coordinate corresponding to rotations about an axis of symmetry, and must therefore vanish on this axis, which ensures that condition (a) is obeyed. The same assumption can be used to motivate condition (a) in the higher dimensional case. Of course, this is not the only way in which condition (a) can be satisfied, so this theorem has wider applicability than just metrics with an axis of rotational symmetry.

If the conditions of this theorem are met then the coordinates $y^{1}$ and $y^{2}$ can be chosen in one of the orthogonal surfaces and then extended along the integral curves of the Killing vector fields. ${ }^{3}$ In this coordinate system, the vectors $\partial / \partial y^{i}$ are orthogonal to $\partial / \partial x^{j}$. If it is further assumed that the Killing vector fields are orthogonal to each other then the metric must take the form

$$
d s^{2}=\sum_{i=1}^{D-2} \epsilon_{i} e^{2 U_{i}}\left(d x^{i}\right)^{2}+g_{a b} d y^{a} d y^{b},
$$

where $a$ and $b$ take the values 1,2 , the metric coefficients are independent of $x^{i}$, and $\epsilon_{i}= \pm 1$ according to whether $\xi_{(i)}$ is spacelike or timelike.

The final step is to use the freedom to perform coordinate transformations on $y^{a}$. Locally it is always possible to choose coordinates such that

$$
g_{a b} d y^{a} d y^{b}=e^{2 C} d Z d \bar{Z},
$$

where $Z$ and $\bar{Z}$ are complex conjugate coordinates if the transverse space is spacelike, and independent real coordinates if it is timelike. ${ }^{4}$ The function $C$ is independent of $x^{i}$.

\section{B. Solving the Einstein equations}

We have shown that any $D$-dimensional metric that admits $D-2$ orthogonal commuting Killing vector fields can be written locally in the form

$$
d s^{2}=\sum_{i=1}^{D-2} \epsilon_{i} e^{2 U_{i}}\left(d x^{i}\right)^{2}+e^{2 C} d Z d \bar{Z}
$$

where $U_{i}$ and $C$ are functions of $Z$ and $\bar{Z}$ only, and $\epsilon_{i}=$ \pm 1 . The summation convention will not be used for indices $i, j, \ldots$.

The components of the curvature tensors of this line element are calculated in Appendix A. The vacuum Einstein equations read $R_{\mu \nu}=0$. The $i j$ component gives

$$
\partial_{Z}\left[\exp \left(\sum_{j} U_{j}\right) \partial_{\bar{Z}} U_{i}\right]+\partial_{\bar{Z}}\left[\exp \left(\sum_{j} U_{j}\right) \partial_{Z} U_{i}\right]=0 .
$$

Summing this equation over $i$ yields

$$
\partial_{Z} \partial_{\bar{Z}} \exp \left(\sum_{j} U_{j}\right)=0
$$

which has the general solution

\footnotetext{
${ }^{3}$ It is necessary to assume that the $\xi_{(i)}$ are non-null at this point. "The term "Weyl solution" is usually reserved for static solutions (i.e., a spacelike transverse space) but we adopt a more general usage here.
} 


$$
\sum_{j} U_{j}=\log (w(Z)+\tilde{w}(\bar{Z})),
$$

where $\tilde{w}=\bar{w}$ if $Z$ and $\bar{Z}$ are complex conjugate, but $w$ and $\tilde{w}$ are independent real functions if $Z$ and $\bar{Z}$ are real coordinates. Substituting Eq. (2.6) into Eq. (2.4) yields

$$
2(w+\tilde{w}) \partial_{Z} \partial_{\bar{Z}} U_{i}+\partial_{Z} w \partial_{\bar{Z}} U_{i}+\partial_{\bar{Z}} \tilde{w} \partial_{Z} U_{i}=0 .
$$

If $w$ is nonconstant then $R_{Z Z}=0$ can be rearranged to give

$$
\partial_{Z} C=\frac{\sum_{i} \partial_{Z}^{2} U_{i}}{\sum_{i} \partial_{Z} U_{i}}+\frac{1}{2} \sum_{i} \partial_{Z} U_{i}-\frac{\sum_{i<j} \partial_{Z} U_{i} \partial_{Z} U_{j}}{2 \sum_{i} \partial_{Z} U_{i}} .
$$

A similar equation arises from $R_{\bar{Z} \bar{Z}}=0$ (assuming that $\tilde{w}$ is nonconstant):

$$
\partial_{\bar{Z}} C=\frac{\sum_{i} \partial_{\bar{Z}}^{2} U_{i}}{\sum_{i} \partial_{\bar{Z}} U_{i}}+\frac{1}{2} \sum_{i} \partial_{\bar{Z}} U_{i}-\frac{\sum_{i<j} \partial_{\bar{Z}} U_{i} \partial_{\bar{Z}} U_{j}}{2 \sum_{i} \partial_{\bar{Z}} U_{i}} .
$$

The first two terms of these equations be integrated immediately, using Eq. (2.6) to give

$$
C=\frac{1}{2} \log \left(\partial_{Z} w \partial_{\bar{Z}} \tilde{w}\right)+\nu,
$$

where

$$
\begin{aligned}
& \partial_{Z} \nu=-\frac{w+\tilde{w}}{\partial_{Z} w} \sum_{i<j} \partial_{Z} U_{i} \partial_{Z} U_{j}, \\
& \partial_{\bar{Z}} \nu=-\frac{w+\tilde{w}}{\partial_{\bar{Z}} \tilde{w}} \sum_{i<j} \partial_{\bar{Z}} U_{i} \partial_{\bar{Z}} U_{j} .
\end{aligned}
$$

The integrability condition for $\nu$ is

$$
\partial_{Z} \partial_{\bar{Z}} \nu=\partial_{\bar{Z}} \partial_{Z} \nu
$$

It is straightforward to check that this equation is indeed satisfied by using Eqs. (2.6) and (2.7). These equations also ensure that the remaining Einstein equation $R_{Z \bar{Z}}=0$ is satisfied.

The only assumptions made above were that $w(Z)$ and $\tilde{w}(\bar{Z})$ are nonconstant. The special cases when one (or both) of these functions is constant will be dealt with in Sec. II D. With this exception, it has been demonstrated that the most general solution of the $D$-dimensional Einstein equations that admits $D-2$ orthogonal commuting Killing vector fields takes the form (2.3), where $U_{i}$ are solutions of Eq. (2.7) subject to the constraint (2.6), and $C$ is given by Eq. (2.10). The function $\nu$ in this equation is given by integrating Eqs. (2.11) and (2.12).
The constraint (2.6) can be eliminated by using it to express, say, $U_{1}$ in terms of $U_{2} \ldots U_{D-2}$. If this is done then $C$ can be written

$$
C=\frac{1}{2} \log \left(\partial_{Z} w \partial_{\bar{Z}} \tilde{w}\right)-\sum_{i>1} U_{i}+\gamma,
$$

where $\gamma$ is given by integrating

$$
\begin{aligned}
& \partial_{Z} \gamma=\frac{w+\tilde{w}}{\partial_{Z} w}\left[\sum_{i>1}\left(\partial_{Z} U_{i}\right)^{2}+\sum_{1<i<j} \partial_{Z} U_{i} \partial_{Z} U_{j}\right], \\
& \partial_{\bar{Z}} \gamma=\frac{w+\tilde{w}}{\partial_{\bar{Z}} \tilde{w}}\left[\sum_{i>1}\left(\partial_{\bar{Z}} U_{i}\right)^{2}+\sum_{1<i<j} \partial_{\bar{Z}} U_{i} \partial_{\bar{Z}} U_{j}\right] .
\end{aligned}
$$

\section{Relation to Laplace's equation}

Since $w$ and $\tilde{w}$ have been assumed nonconstant, it is legitimate to perform a coordinate transformation from $Z$ and $\bar{Z}$ to $w(Z)$ and $\tilde{w}(\bar{Z})$. In four dimensions, these are referred to as "Weyl's canonical coordinates" [1]. This gives

$$
d s^{2}=\sum_{i} \epsilon_{i} e^{2 U_{i}}\left(d x^{i}\right)^{2}+e^{2 v} d w d \tilde{w} .
$$

This coordinate transformation is conformal. Equations (2.7), (2.11), and (2.12) are conformally invariant so the transformation just replaces $\partial_{Z}$ by $\partial \equiv \partial_{w}$ and $\partial_{\bar{Z}}$ by $\bar{\partial} \equiv \partial_{\tilde{w}}$. Then the solution is determined by the following equations:

$$
\begin{gathered}
\sum_{i} U_{i}=\log (w+\tilde{w}), \\
2(w+\tilde{w}) \partial \bar{\partial} U_{i}+\partial U_{i}+\bar{\partial} U_{i}=0, \\
\partial \nu=-(w+\tilde{w}) \sum_{i<j} \partial U_{i} \partial U_{j},
\end{gathered}
$$

$$
\bar{\partial} \nu=-(w+\tilde{w}) \sum_{i<j} \bar{\partial} U_{i} \bar{\partial} U_{j}
$$

If one prefers to eliminate the constraint (2.18) then the metric takes the form

$$
\begin{aligned}
d s^{2}= & \exp \left(-2 \sum_{i>1} U_{i}\right)\left[e^{2 \gamma} d w d \tilde{w}+\epsilon_{1}(w+\tilde{w})^{2}\left(d x^{1}\right)^{2}\right] \\
& +\sum_{i>1} \epsilon_{i} e^{2 U_{i}}\left(d x^{i}\right)^{2}
\end{aligned}
$$

with $\gamma$ determined by

$$
\partial \gamma=(w+\tilde{w})\left[\sum_{i>1}\left(\partial U_{i}\right)^{2}+\sum_{1<i<j} \partial U_{i} \partial U_{j}\right],
$$




$$
\bar{\partial} \gamma=(w+\tilde{w})\left[\sum_{i>1}\left(\bar{\partial} U_{i}\right)^{2}+\sum_{1<i<j} \bar{\partial} U_{i} \bar{\partial} U_{j}\right] .
$$

If $Z$ and $\bar{Z}$ are complex conjugate coordinates then, as mentioned above, one must take $\tilde{w}=\bar{w}$. Introduce real coordinates $(r, z)$ by $w=r+i z$, so the canonical form of the metric is

$$
d s^{2}=\sum_{i} \epsilon_{i} e^{2 U_{i}}\left(d x^{i}\right)^{2}+e^{2 \nu}\left(d r^{2}+d z^{2}\right) .
$$

Equation (2.19) then takes the form

$$
\frac{\partial^{2} U_{i}}{\partial r^{2}}+\frac{1}{r} \frac{\partial U_{i}}{\partial r}+\frac{\partial^{2} U_{i}}{\partial z^{2}}=0
$$

which is just Laplace's equation in three-dimensional flat space with metric

$$
d s^{2}=d r^{2}+r^{2} d \theta^{2}+d z^{2} .
$$

The function $U_{i}$ is independent of the (unphysical) coordinate $\theta$, i.e., it is axisymmetric. The solution is therefore specified by $D-3$ independent axisymmetric solutions of Laplace's equation in three-dimensional flat space. There are only $D-3$ independent $U_{i}$ because of the constraint (2.18), which can now be written

$$
\sum_{i} U_{i}=\log r+\text { const, }
$$

where the constant term can be freely adjusted by rescaling the coordinates $x^{i}$. Note that $\log r$ is the solution of Laplace's equation that describes the Newtonian potential produced by an infinite rod of zero thickness lying along the $z$ axis, with constant mass $1 / 2$ per unit length (in units $G=1$ ). The solutions for $U_{i}$ can also be thought of as Newtonian potentials produced by certain sources, so the constraint (2.28) states that these sources must add up to give an infinite rod. Note that the solution is completely determined by these sources. The sources for $U_{i}$ will sometimes be referred to as the sources for $x^{i}$.

For $D=4$, the metric (2.22) can be brought to the standard form of Eq. (1.1) by taking $\epsilon_{1}=-\epsilon_{2}=1, x^{2}=t, x^{1}$ $=\phi / 2$, and $U_{2}=U$. However, this form obscures the symmetry between $x^{1}$ and $x^{2}$, and hides the fact that solutions which have different sources for $U$ may actually be equivalent under interchange of $t$ and $\phi$. We will illustrate this point with an example in Sec. III B.

If $w$ and $\tilde{w}$ are real coordinates then they can be viewed as advanced and retarded null coordinates. Introduce new coordinates $(t, r)$ defined by $w=r+t, \quad \tilde{w}=r-t$. Then Eq. (2.18) becomes

$$
-\frac{\partial^{2} U_{i}}{\partial t^{2}}+\frac{\partial^{2} U_{i}}{\partial r^{2}}+\frac{1}{r} \frac{\partial U_{i}}{\partial r}=0,
$$

which is just the wave equation in a three-dimensional flat spacetime with metric

$$
d s^{2}=-d t^{2}+d r^{2}+r^{2} d \theta^{2},
$$

with the function $U_{i}$ independent of the unphysical coordinate $\theta$. The solution is specified by $D-2$ axisymmetric solutions of the wave equation in three-dimensional flat spacetime but only $D-3$ of these are independent because the constraint (2.18) states that these solutions must add up to the static solution describing a point source at the origin of polar coordinates.

\section{Special classes of solutions}

It was assumed above that $w(Z)$ and $\tilde{w}(\bar{Z})$ are nonconstant, but there is the possibility that one or both of these quantities is constant. These solutions are discussed in Appendix B. They will be referred to as special Weyl solutions to distinguish them from those of the previous sections, which will be referred to as generic Weyl solutions. For $D$ $=4$ the special solutions are either flat space or $p p$ waves. The interpretation of the solutions in $D>4$ is unclear, so it might be interesting to investigate them further. In $D=5$, most of the static special solutions appear to be nakedly singular but there may be exceptions.

In the rest of the paper we will consider only static $(\tilde{w}$ $=\bar{w}$ ) Weyl solutions of the generic class.

\section{WEYL FORM OF KNOWN SOLUTIONS}

Generic Weyl metrics are characterized by axisymmetric harmonic functions in three-dimensional flat space. If such functions are regarded as Newtonian potentials produced by axisymmetric sources, then Weyl solutions can be completely characterized by these sources. In order to identify the types of sources that might be relevant in attempting to find interesting new solutions, we study the Weyl form for some known physically relevant metrics.

\section{A. Flat space}

We study in Appendix $\mathrm{C}$ the circumstances under which the metric (2.3) is flat. There are three possibilities. This first is trivially given by taking all of the functions $U_{i}$ to be constant. $^{5}$ The second possibility corresponds to all but one of the functions $U_{i}$ being constant, and one of the $U_{i}$ (say, $U_{1}$ ) being the potential of an infinite rod along the $z$ axis, $U_{1}=\log r+$ const. The metric in this case can be brought to the form (see Appendix C)

$$
d s^{2}=\epsilon_{1} \xi^{2}\left(d x^{1}\right)^{2}+\sum_{i=2}^{D-2} \epsilon_{i}\left(d x^{i}\right)^{2}+d \xi^{2}+d \eta^{2},
$$

so if $\epsilon_{1}=+1$ then $x^{1}$ is an azimuthal angle and if $\epsilon_{1}=-1$ then $x^{1}$ is a Rindler (boost) time coordinate.

\footnotetext{
${ }^{5}$ This can be regarded as belonging to the special Weyl class.
} 
The third possibility corresponds to all but two of the functions $U_{i}$ being constant, with one of the remaining two being the potential of a semi-infinite rod along the $z \geqslant a$ portion of the $z$ axis (for some $a$ ), and the other being the potential of a semi-infinite rod along the $z \leqslant a$ portion of the $z$ axis (see Fig. 1).

In terms of $w$,

$$
\begin{aligned}
& U_{1}=\log |\operatorname{Re} \sqrt{a \pm i w}|+\text { const } \\
& U_{2}=\log |\operatorname{Re} \sqrt{-a \mp i w}|+\text { const. }
\end{aligned}
$$

Writing these in terms of $r, z$ gives

$$
\begin{aligned}
& U_{1}=\frac{1}{2} \log \left[a \mp z+\sqrt{(a \mp z)^{2}+r^{2}}\right]+\text { const, } \\
& U_{2}=\frac{1}{2} \log \left[-a \pm z+\sqrt{(-a \pm z)^{2}+r^{2}}\right]+\text { const. }
\end{aligned}
$$

The upper sign choice corresponds to $U_{1}$ being the potential of a semi-infinite rod $z \geqslant a$ and $U_{2}$ being that of a semiinfinite $\operatorname{rod} z \leqslant a$. The lower sign choice corresponds to the source for $U_{1}$ being a semi-infinite $\operatorname{rod} z \leqslant-a$ and the source for $U_{2}$ a semi-infinite $\operatorname{rod} z \geqslant-a$. The rods are all on the $z$ axis, have zero thickness, and mass $1 / 2$ per unit length. The metric in this case can be brought to the form (see Appendix C)

$d s^{2}=\epsilon_{1} \xi^{2}\left(d x^{1}\right)^{2}+\epsilon_{2} \eta^{2}\left(d x^{2}\right)^{2}+\sum_{i=3}^{D-2} \epsilon_{i}\left(d x^{i}\right)^{2}+d \xi^{2}+d \eta^{2}$,

so the coordinates $x^{1}$ and $x^{2}$ are azimuthal angles or Rindler time coordinates according to the signs of $\epsilon_{1}$ and $\epsilon_{2}$.

\section{B. The Schwarzschild solution}

The $D$-dimensional Schwarzschild solution has isometry group $\mathbf{R} \times O(D-1)$. To write it in Weyl form, $D-2$ orthogonal commuting Killing vector fields are required. For the Schwarzschild solution, this occurs only for $D=4,5$. Hence only the four and five-dimensional Schwarzschild solutions can be written in Weyl form. The Weyl form of the four-dimensional Schwarzschild solution is well known so it will be discussed only briefly here.

For $D=4$, a generic Weyl solution can be converted to the form of Eq. (1.1) as described in Sec. II C. For the Schwarzschild metric, the function $U$ is given by

$$
U=-\frac{1}{2} \log \left[\frac{M-z+\sqrt{(M-z)^{2}+r^{2}}}{-M-z+\sqrt{(M+z)^{2}+r^{2}}}\right],
$$

where $M$ is the Schwarzschild mass parameter. $U \equiv U_{1}$ is the potential of a finite rod along the $-M \leqslant z \leqslant M$ portion $^{6}$ of the

\footnotetext{
${ }^{6}$ One is free to shift the rod to any position on the $z$ axis with a transformation $z \rightarrow z+a$.
}

$z$ axis. The rod has vanishing thickness and mass $1 / 2$ per unit length. It follows from the constraint (2.28) that the function $U_{2}$ must be the potential produced by semi-infinite rods $z$ $\geqslant M$ and $z \leqslant-M$. These sources are depicted in Fig. 2(a).

Note that our approach makes clear the nature of the solution of the $D=4$ Weyl class (1.1) that is obtained by taking two semi-infinite rod sources for $U$. In our approach it simply corresponds to interchanging $x^{1}$ and $x^{2}$, i.e., interchanging the time $t$ and azimuthal angle $\phi$ coordinates. This gives a four-dimensional analogue of the expanding KK bubble of [19], and describes the decay of the $D=4 \mathrm{KK}$ vacuum $M^{1,2} \times S^{1}[26]$.

The five-dimensional Schwarzschild metric can be written in Schwarzschild coordinates as

$$
\begin{aligned}
d s^{2}= & -\left(1-\frac{\mu}{R^{2}}\right) d t^{2}+\left(1-\frac{\mu}{R^{2}}\right)^{-1} d R^{2}+R^{2} d \theta^{2} \\
& +R^{2} \sin ^{2} \theta d \phi^{2}+R^{2} \cos ^{2} \theta d \psi^{2},
\end{aligned}
$$

where $0 \leqslant \theta \leqslant \pi / 2$, and $\phi \sim \phi+2 \pi, \quad \psi \sim \psi+2 \pi$. There are clearly three orthogonal commuting Killing vector fields. Take $x^{1}=t, \quad x^{2}=\phi, \quad x^{3}=\psi$ with $\epsilon_{1}=-1, \quad \epsilon_{2}=\epsilon_{3}=1$. Then one can read off

$$
e^{U_{1}}=\left(1-\frac{\mu}{R^{2}}\right)^{1 / 2}, \quad e^{U_{2}}=R \sin \theta, \quad e^{U_{3}}=R \cos \theta .
$$

The constraint (2.28) gives

$$
U_{1}+U_{2}+U_{3}=\log r
$$

where the constant term has been absorbed into the normalization of $r$. This equation implies

$$
r=\frac{1}{2}\left(1-\frac{\mu}{R^{2}}\right)^{1 / 2} R^{2} \sin 2 \theta .
$$

To bring the metric to Weyl form, it is necessary to define $z$ such that

$$
d r^{2}+d z^{2} \equiv d w d \bar{w} \propto\left(1-\frac{\mu}{R^{2}}\right)^{-1} d R^{2}+R^{2} d \theta^{2} .
$$

Substituting the ansatz $z=g(R) \cos 2 \theta$ into this equation then uniquely determines $g(R)$, giving

$$
z=\frac{1}{2}\left(1-\frac{\mu}{2 R^{2}}\right) R^{2} \cos 2 \theta .
$$

It also possible to read off $\gamma$ :

$$
e^{2 \gamma}=\frac{1}{4}\left(1-\frac{\mu}{R^{2}}+\frac{\mu^{2}}{4 R^{4}} \cos ^{2} 2 \theta\right)^{-1} R^{2} \sin ^{2} 2 \theta
$$




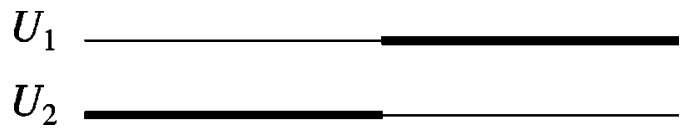

FIG. 1. Sources for the harmonic functions of one of the Weyl forms of flat space. The thin lines denote the $z$ axis and the thick lines denote thin rods along this axis. The left and right ends of the figure are to be interpreted as $z=-\infty$ and $z=+\infty$ respectively. The sources for $U_{1}$ and $U_{2}$ are semi-infinite rods of mass $1 / 2$ per unit length. The $U_{1}$ source lies along $z \geqslant a$ and the $U_{2}$ source along $z$ $\leqslant a$ for some $a$. In the classification of Sec. IV B, this is a class 0 solution.

It remains to write the functions $U_{i}$ in terms of $r$ and $z$. To do this, let $X=e^{U_{2}}$ and $Y=e^{U_{3}}$. Equations (3.11) and (3.13) can then be written as

$$
\begin{aligned}
& r^{2}=\left(1-\frac{\mu}{X^{2}+Y^{2}}\right) X^{2} Y^{2}, \\
& z=\frac{1}{2}\left(1-\frac{\mu}{2\left(X^{2}+Y^{2}\right)}\right)\left(Y^{2}-X^{2}\right),
\end{aligned}
$$

which can be rearranged to give

$$
\begin{aligned}
& 2 Y^{4}-(\mu+4 z) Y^{2}-2 r^{2}=0, \\
& 2 X^{4}-(\mu-4 z) X^{2}-2 r^{2}=0 .
\end{aligned}
$$

Solving these yields

$$
\begin{aligned}
& U_{2}=\frac{1}{2} \log \left[\frac{\mu}{4}-z+\sqrt{\left(\frac{\mu}{4}-z\right)^{2}+r^{2}}\right], \\
& U_{3}=\frac{1}{2} \log \left[\frac{\mu}{4}+z+\sqrt{\left(\frac{\mu}{4}+z\right)^{2}+r^{2}}\right] .
\end{aligned}
$$

$\gamma$ can be written in terms of $w$ and $\bar{w}$ to check that Eqs. (2.23) and (2.24) are obeyed. The explicit expression will not be written out here since it can be obtained as a special case of more general expressions given later in this paper. $U_{2}$ is the potential of a semi-infinite rod with vanishing thickness and mass $1 / 2$ per unit length positioned along the $z$ axis at $z \geqslant \mu / 4 . U_{3}$ is the potential of an identical rod along the $z$ axis at $z \leqslant-\mu / 4$. The function $U_{1}$ is obtained from Eq. (3.10) and is the potential of a rod along the $-\mu / 4 \leqslant z$ $\leqslant \mu / 4$ portion of the $z$ axis, again with vanishing thickness and mass $1 / 2$ per unit length. See Fig. 2(b). Note that the source corresponding to the time coordinate is a finite rod for both the $D=4$ and the $D=5$ Schwarzschild solutions.

Black branes. For $D>5$, the $D$-dimensional Schwarzschild solution is not a generalized Weyl solution. However, the black branes obtained by taking products of the $D=4$ or $D=5$ Schwarzschild solution with flat space are easily seen to be Weyl solutions. The functions $U_{i}$ associated with the flat directions are all constant, and those associated with the Schwarzschild directions can be read off from the results of this section.

\section{Other four-dimensional solutions}

Other physically relevant four-dimensional Weyl solutions are:

The Israel-Khan solutions [27]. These describe finitely many collinear black holes in static equilibrium. The forces holding them apart arise from conical deficits in the form of struts between the black holes, or cosmic strings extending to infinity. If the metric is written in the Weyl form (1.1) then the sources for $U$ are finite rods along the $z$ axis. The rods have zero thickness, mass $1 / 2$ per unit length, and do not intersect. The length of each rod determines the mass of the corresponding black hole. If one considers infinitely many such rods of equal length and equally spaced then one can eliminate the need for conical singularities and obtain a solution describing an infinite line of black holes [22].

The $C$ metric [10]. The $C$ metric is a four-dimensional metric that describes two black holes accelerating apart. The force for the acceleration is provided by a conical deficit, which occurs either in the form of a strut between the two black holes or as a cosmic string stretching off to infinity from each hole. The Weyl form of the $C$ metric was obtained in $[28,29]$. The function $U$ is the potential of a finite rod and a semi-infinite rod, which do not intersect. Both rods lie along the $z$ axis, have zero thickness and have mass $1 / 2$ per unit length [see Fig. 3(a) with $U_{1}=U$ ]. The finite rod corresponds to one of the black holes and the semi-infinite rod is responsible for the acceleration field (the second black hole lies beyond an acceleration horizon, so it is not apparent in the Weyl coordinates). Adding further finite rod sources to $U$ results in a metric describing multiple accelerating black holes connected by conical deficits [30].

\section{NEW SOLUTIONS}

The sources for the solutions discussed in the previous section are all rods of zero thickness and mass $1 / 2$ per unit length. The rods are on the $z$ axis and can be finite, semiinfinite or infinite. More general sources typically give rise to naked curvature singularities on the axis of symmetry. Hence, in attempting to find interesting new Weyl solutions we will consider only sources of this form. The examples of the previous section allow us to make some general observations that are useful when analyzing a solution given its set of sources.

\section{A. General comments}

The constraint (2.28) is very restrictive: It states that the sources for the different $U_{i}$ 's must add up to an infinite rod along the $z$ axis, again with mass $1 / 2$ per unit length. Assuming that only finitely many rods are present, it follows that either one of the $U_{i}$ 's has semi-infinite rod sources which extends to $z=\infty$ and $z=-\infty$, or there is one $U_{i}$ with a semiinfinite rod source which extends to $z=\infty$ and another with a semi-infinite rod source which extend to $z=-\infty$. All of the other $U_{i}$ 's must have bounded sources consisting of a finite number of finite rods.

If the source for $U_{i}$ is bounded (i.e., involves only finitely many finite rods) then $U_{i}$ must approach a constant far from 


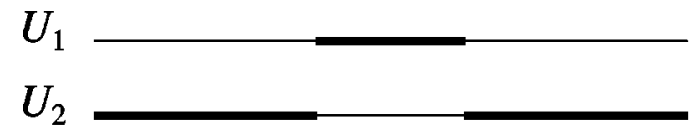

(a)

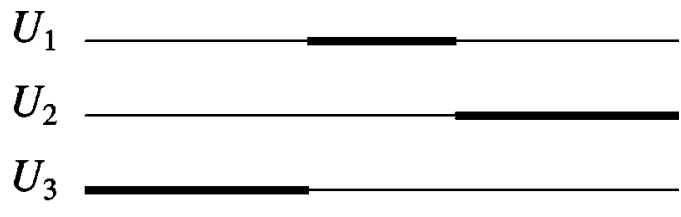

(b)

FIG. 2. Sources for the (a) four dimensional and (b) five dimensional Schwarzschild solutions. The black hole interpretation requires that $x^{1}$ is the timelike coordinate. If in (a) $x^{2}$ is the timelike coordinate then this describes an expanding bubble in the $M^{1,2} \times S^{1}$ vacuum. If both $x^{1}$ and $x^{2}$ are spacelike then this describes a static KK $S^{2}$ bubble (when a trivial time direction is added). If in (b) $x^{2}$ (or $x^{3}$ ) corresponds to time, then it describes an expanding bubble in the $M^{1,3} \times S^{1}$ vacuum. If $x^{1}, x^{2}$, and $x^{3}$ are all spatial coordinates, then it describes an $S^{3}$ bubble. In the classification of Sec. IV B, these solutions are class I.

the source. It follows that $x^{i}$ must be a flat direction in the asymptotic metric. An example of this is provided by the Schwarzschild metrics, for which the source corresponding to the time coordinate is a finite rod, and the time direction does indeed become flat in the asymptotic region.

Now consider the behavior near the sources. Assume first that $x^{i}$ is a time coordinate. For the $D=4,5$ Schwarzschild solutions, the source for $U_{i}$ is a finite rod and the region near this source corresponds to the event horizon of the black hole. This is also true for the finite rod sources in the $C$ metric and Israel-Khan solutions. The semi-infinite rod source of the $C$ metric corresponds to a horizon that extends to asymptotic infinity - this is an acceleration horizon, which arises because the time coordinate behaves like a boost at asymptotic infinity. It was shown above that flat space can also be written in a Weyl form in which the time coordinate has a semi-infinite rod source. This source also corresponds to an acceleration horizon, arising because the time coordinate is a boost, i.e., the Rindler time coordinate. To summarize: finite rod sources for the time coordinate correspond to event horizons in spacetime, and semi-infinite rod sources correspond to acceleration horizons.

The case in which $x^{i}$ is a spatial coordinate can be understood by Euclideanizing some of the metrics discussed above. For the Euclidean Schwarzschild solution, the finite rod source corresponds to the "bolt" where the Euclidean time direction closes off smoothly, provided it is identified with a suitable period. A similar interpretation holds for Euclideanized Rindler space, with the only difference being that in the former case, the bolt is finite in extent (it is an $S^{2}$ in $D=4$ ) whereas in the latter case it extends to infinity (it is $R^{2}$ in $\left.D=4\right)$. These features also occur for the other examples above. In conclusion: rod sources for a spatial coordinate $x^{i}$ correspond to "bolts:" fixed-point sets of the orbits of $\partial / \partial x^{i}$. If the source for $x^{i}$ extends to infinity, then the bolt will also extend to infinity, corresponding to an axis of rotational symmetry with $x^{i}$ acting as the azimuthal angle.

In order to avoid a conical singularity, $x^{i}$ has to be periodically identified with a particular period determined by the sources. If there is more than one source then there will be several bolts, and the appropriate periods for $x^{i}$ at each bolt may differ. In this case, conical singularities will result. This occurs for the $C$ metric and Israel-Khan solutions as well as those of [30].
A final fact useful when analyzing Weyl solutions immediately follows from the above discussion. Let $x^{i}$ be a spatial coordinate with a single finite rod source. Then $x^{i}$ has to be identified with a certain period in order to avoid a conical singularity at the bolt corresponding to the source. Moreover, $x^{i}$ is a flat coordinate in the asymptotic region. It follows that $x^{i}$ is most naturally interpreted as parametrizing a KK circle at infinity. If there is more than one finite rod source then it might no longer be possible to remove all conical singularities by identifying $x^{i}$ but one would probably still wish to minimize the number of singularities by an appropriate identification. Hence: if a spatial coordinate $x^{i}$ has only finite rod sources then it can be interpreted as a KK coordinate in the asymptotic region. The rod sources, where the $\mathrm{KK}$ circle shrinks to zero size, appear as singularities in the dimensionally reduced description.

Now, since at most two of the $U_{i}$ 's have sources extending to infinity, it follows that at least $D-4$ of the $x^{i}$, s will have bounded sources. If one of these is the time coordinate then there will be at least $D-5$ spatial coordinates with bounded sources so the asymptotic metric will have at least $D-5$ compactified flat directions. It follows that no $D>5$ Weyl solution can be asymptotically flat (in the global sense) if it has sources of the form being considered here.

\section{B. Classification}

A solution will be said to be of class $n$ if it has $n$ finite rod sources (as well as a suitable number of infinite, or semiinfinite rod sources).

We make no distinction between metrics related by Wick rotation, so any of the $x^{i}$ can be chosen as the time direction. The first few classes are:

Class 0 . If there are no finite rod sources then the sources must be either an infinite rod, or two semi-infinite rods (Fig. 1). It was shown above that the metric is flat in both of these cases, so flat space is the only class 0 solution.

Class $I$. In this class, there is a single finite rod so the other sources must be two semi-infinite rods (Fig. 2). There are two ways that these sources can be distributed amongst the $U_{i}$ 's. (a) $U_{1}$ has a finite rod source and $U_{2}$ has both semi-infinite rod sources. The other $U_{i}$ are constant. This is the four-dimensional Schwarzschild solution (times some flat directions if $D>4$ ). (b) $U_{1}$ has a finite rod source, and $U_{2}$ 


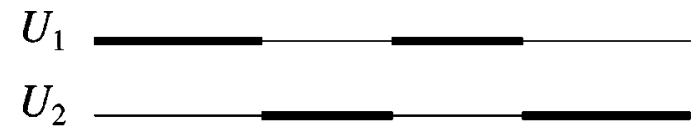

(a)

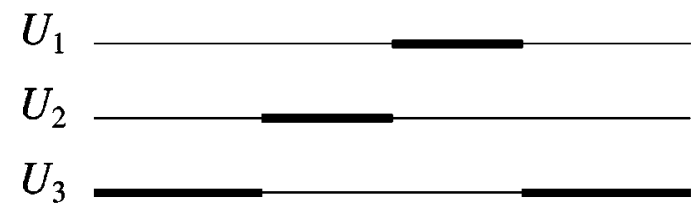

(c)

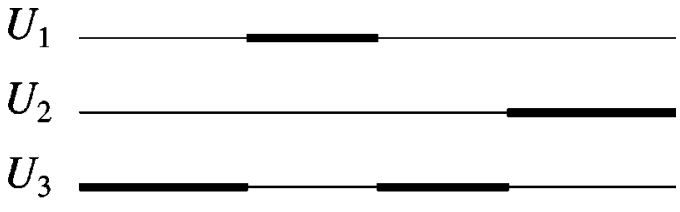

(b)

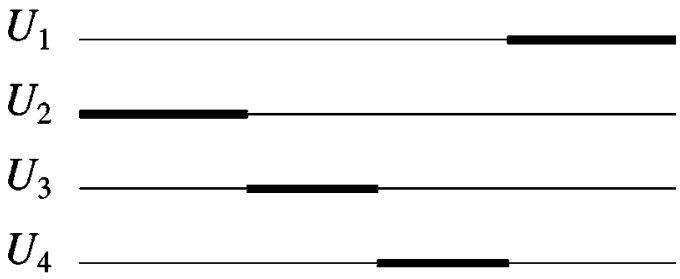

(d)

FIG. 3. Sources for (a) $C$ metric, (b) black ring, (c) black hole plus KK bubble, and (d) black string and KK bubble. Note that the sources for the $U_{i}$ 's have to add up to an infinite rod. In the classification of Sec. IV B, these solutions are class II.

and $U_{3}$ have semi-infinite rod sources. The other $U_{i}$ are constant. This is the five-dimensional Schwarzschild solution (times some flat directions if $D>5$ ).

Class II. The sources are two finite rods $a_{3} \leqslant z \leqslant a_{2}$ and $a_{2} \leqslant z \leqslant a_{1}$ and two semi-infinite rods $z \geqslant a_{1}$ and $z \leqslant a_{3}$ (Fig. $3)$. There are four ways to distribute these sources amongst the $U_{i}$ 's. Flat dimensions (corresponding to constant $U_{i}$ ) will be neglected. (a) $U_{1}$ has a semi-infinite rod source and a finite rod source, as does $U_{2}$. This gives the four-dimensional $C$ metric. (b) $U_{1}$ has a finite rod source, $U_{2}$ has a semiinfinite rod, and $U_{3}$ has a finite rod and a semi-infinite rod. This is a new "black ring" solution that will be discussed in Sec. IV C. (c) $U_{1}$ and $U_{2}$ have the finite rods as sources, and $U_{3}$ has both semi-infinite rods as its sources. This is a new $D=5$ solution describing a superposition of a black hole with a Kaluza-Klein bubble. (d) $U_{1}$ and $U_{2}$ have the semiinfinite rods as sources, and $U_{3}$ and $U_{4}$ have the finite rods as sources. This is a new $D=6$ solution describing the superposition of a black string with a Kaluza-Klein bubble. Solutions (c) and (d) will be discussed in Secs. IV E and IV F respectively.

The $D=4$ class $n$ solutions for $n>2$ have all been discussed before. If $n$ is odd, $n=2 k-1$, then the solution is an Israel-Khan solution describing $k$ black holes. If $n$ is even, $n=2 k$, then the solution is a generalization of the $C$ metric of the form discussed in [30] and describes $k$ accelerating black holes on each side of an acceleration horizon. Conical singularities are present in both cases.

A solution in a given class can be reduced to a solution of a lower class by either contracting to zero size or expanding to infinity one of its finite rods. The limits that must be taken to recover a given solution can be easily deduced by looking at the diagrams for sources in Figs. 1, 2, and 3. For example, from Fig. 3(a) we easily see that the $C$ metric has a limit where one recovers the $D=4$ Schwarzschild solution, Fig. 2(a), by taking to infinity the leftmost end point of the $x^{2}$ rod. Effectively, this amounts to removing the acceleration horizon from the metric, the well-known limit where the acceleration of the black hole is set to zero. The $C$ metric also has several limits where flat (Rindler) space, Fig. 1, is recovered. The II(b) solution that below will be interpreted as a black ring, Fig. 3(b), similarly reduces to either a $D=5$ black hole [Fig. 2(b)], or a black string obtained as the product of the $D=4$ black hole [Fig. 2(a)] and a flat spatial direction.

\section{The black ring}

In [24] an unconventional neutral limit for the KK charged $C$ metric ( [12] dualized to have electric charge) was taken. The resulting metric was interpreted as describing a pair of KK bubbles being accelerated apart by a conical singularity. We now show that this metric has a less exotic interpretation if one Wick rotates it to give

$$
\begin{aligned}
d s^{2}= & -\frac{F(x)}{F(y)} d t^{2}+\frac{1}{A^{2}(x-y)^{2}}\left[F ( x ) \left(\left(y^{2}-1\right) d \psi^{2}\right.\right. \\
& \left.\left.+\frac{F(y)}{y^{2}-1} d y^{2}\right)+F(y)^{2}\left(\frac{d x^{2}}{1-x^{2}}+\frac{1-x^{2}}{F(x)} d \phi^{2}\right)\right],
\end{aligned}
$$

where

$$
F(\xi)=1-\mu \xi
$$

The parameters $\mu$ and $A$ will be taken to lie in the range 0 $\leqslant \mu \leqslant 1, \quad A>0$, the coordinate $x$ in the range $-1 \leqslant x \leqslant 1$ 
and the coordinate $y$ in the range $y \leqslant-1$. This metric clearly has three orthogonal commuting Killing vector fields so it is a Weyl solution. Choosing $t=x^{1}, \psi=x^{2}$, and $\phi=x^{3}$, the functions $U_{i}$ are given by

$$
\begin{gathered}
e^{2 U_{1}}=\frac{F(x)}{F(y)}, \\
e^{2 U_{2}}=\frac{\left(y^{2}-1\right) F(x)}{A^{2}(x-y)^{2}}, \\
e^{2 U_{3}}=\frac{\left(1-x^{2}\right) F(y)^{2}}{A^{2}(x-y)^{2} F(x)} .
\end{gathered}
$$

In order to identify the sources that produce this solution it is necessary to work with the coordinates $r, z$. From Eq. (2.28), it follows that

$$
r=\frac{\alpha}{A^{2}(x-y)^{2}} \sqrt{F(x) F(y)\left(1-x^{2}\right)\left(y^{2}-1\right)},
$$

for some positive constant $\alpha$. The coordinate $z$ is obtained from the requirement

$$
d r^{2}+d z^{2} \propto \frac{F(y)}{1-x^{2}} d x^{2}+\frac{F(x)}{y^{2}-1} d y^{2},
$$

which yields

$$
\frac{\partial z}{\partial x}= \pm \sqrt{\frac{\left(y^{2}-1\right) F(y)}{\left(1-x^{2}\right) F(x)}} \frac{\partial r}{\partial y},
$$

and

$$
\frac{\partial z}{\partial y}=\mp \sqrt{\frac{\left(1-x^{2}\right) F(x)}{\left(y^{2}-1\right) F(y)}} \frac{\partial r}{\partial x} .
$$

These equations can be integrated to give

$$
z=\frac{\alpha(1-x y)(F(x)+F(y))}{2 A^{2}(x-y)^{2}}
$$

up to a choice of sign and an arbitrary additive constant.

In order to write the solution in Weyl form, it is convenient to define

$$
a_{1}=\alpha /\left(2 A^{2}\right), \quad a_{2}=\alpha \mu /\left(2 A^{2}\right), \quad a_{3}=-\alpha \mu /\left(2 A^{2}\right)
$$

and then introduce the following notation $[27,30]$ :

$$
\begin{aligned}
\zeta_{i} & \equiv z-a_{i}, \\
R_{i} & \equiv \sqrt{r^{2}+\zeta_{i}^{2}}, \\
Y_{i j} & \equiv R_{i} R_{j}+\zeta_{i} \zeta_{j}+r^{2} .
\end{aligned}
$$

Expressions for these quantities in terms of $x$ and $y$ are given in Appendix D. Using these expressions, it is easily seen that if one takes $\alpha=A$ then the Weyl form of the metric is given by

$$
\begin{gathered}
e^{2 U_{1}=\frac{R_{3}-\zeta_{3}}{R_{2}-\zeta_{2}},} \\
e^{2 U_{2}=\left(R_{1}-\zeta_{1}\right) / A,} \\
e^{2 U_{3}}=\frac{\left(R_{1}+\zeta_{1}\right)\left(R_{2}-\zeta_{2}\right)}{A\left(R_{3}-\zeta_{3}\right)}, \\
e^{2 \nu}=\frac{1+\mu}{4 A} \frac{Y_{23}}{R_{1} R_{2} R_{3}} \sqrt{\frac{Y_{12}}{Y_{13}}} \sqrt{\frac{R_{2}-\zeta_{2}}{R_{3}-\zeta_{3}}},
\end{gathered}
$$

from which it follows that $U_{1}$ is the Newtonian potential produced by a finite $\operatorname{rod}-\mu /(2 A) \leqslant z \leqslant \mu /(2 A), \quad U_{2}$ is the potential produced by a semi-infinite $\operatorname{rod} z \geqslant 1 /(2 A)$, and $U_{3}$ is the potential produced by a semi-infinite $\operatorname{rod} z \leqslant$ $-\mu /(2 A)$ and a finite $\operatorname{rod} \mu /(2 A) \leqslant z \leqslant 1 /(2 A)$. Note that, for $\mu=1$, these sources reduce to those of the fivedimensional Schwarzschild solution and hence the metric must reduce to the metric of the Schwarzschild solution, so the function $\nu$ for Schwarzschild can be read off from the above.

\section{Analysis of the black ring}

We now explain why the name "black ring" is appropriate by examining the global structure of this solution. To start, consider how the general comments of Sec. IV apply to this solution. The source for $t$ is a finite rod, so the time direction is expected to be asymptotically flat and there should be a horizon present. The coordinates $\phi$ and $\psi$ both have semi-infinite rod sources, so these coordinates should be periodically identified and will have the interpretation of azimuthal angles in the asymptotic metric.

Consider the form of the metric as $y \rightarrow-\infty$. The ty part of the metric becomes

$$
d s_{t y}^{2} \sim F(x)\left(-\frac{1}{\mu|y|} d t^{2}+\frac{\mu}{A^{2}|y|^{3}} d y^{2}\right) .
$$

Performing the coordinate transformation

$$
y=-\frac{4 \mu}{A^{2} Y^{2}}
$$

gives

$$
d s_{t y}^{2} \sim F(x)\left(-\frac{A^{2} Y^{2}}{4 \mu^{2}} d t^{2}+d Y^{2}\right) .
$$

The metric in brackets is just that of Rindler space with acceleration parameter $a=A /(2 \mu)$. The coordinate transformation that takes this to a manifestly flat metric is

$$
X=Y \cosh a t, \quad T=Y \sinh a t,
$$


giving

$$
d s_{t y}^{2} \sim F(x)\left(-d T^{2}+d X^{2}\right) .
$$

Note that the conformal factor $F(x)$ is always positive for $-1 \leqslant x \leqslant 1$. This analysis shows that the leading order part of the ty metric has a nonsingular horizon at $y=-\infty$. If one examines the subleading order terms, one finds that these are also regular there if the same coordinate transformation is made. It is easy to see that the other terms of the metric can also be smoothly extended through this surface which is therefore a regular horizon. The near-horizon metric is

$$
\begin{aligned}
d s^{2} \sim & F(x)\left(-d T^{2}+d X^{2}+A^{-2} d \psi^{2}\right)+\frac{\mu^{2}}{A^{2}}\left(\frac{d x^{2}}{1-x^{2}}\right. \\
& \left.+\frac{1-x^{2}}{F(x)} d \phi^{2}\right),
\end{aligned}
$$

and the metric of a constant $t$ slice through the horizon is

$$
d s^{2}=\frac{1}{A^{2}}\left[F(x) d \psi^{2}+\mu^{2}\left(\frac{d x^{2}}{1-x^{2}}+\frac{1-x^{2}}{F(x)} d \phi^{2}\right)\right] .
$$

Consider now the $x \phi$ part of the metric, which is conformal to

$$
d s_{x \phi}^{2}=\frac{d x^{2}}{1-x^{2}}+\frac{1-x^{2}}{F(x)} d \phi^{2}
$$

Let $x=-\cos \theta$ with $0 \leqslant \theta \leqslant \pi$. This gives ${ }^{7}$

$$
d s_{x \phi}^{2}=d \theta^{2}+\frac{\sin ^{2} \theta}{1+\mu \cos \theta} d \phi^{2} .
$$

In order for this metric to be regular at $\theta=0$ (i.e. $x=-1$ ), it is necessary to identify $\phi$ with period $2 \pi \sqrt{1+\mu}$. For regularity at $\theta=\pi$ (i.e. $x=1$ ), it is necessary to identify $\phi$ with period $2 \pi \sqrt{1-\mu}$. It is therefore not possible to have regularity at both $x=1$ and $x=-1$. If one demands regularity at $x=-1$ then there is a conical singularity at $x=1$ with deficit angle

$$
\delta_{(x=1)}=-2 \pi\left(\sqrt{\frac{1+\mu}{1-\mu}}-1\right)
$$

which is negative so this is really an excess angle. If one demands regularity at $x=1$ then there is a conical singularity at $x=-1$ with deficit angle

\footnotetext{
${ }^{7}$ If $\mu=1$ then the following analysis does not apply, but it is easy to see that the $x \psi \phi$ part of the metric describes a round $S^{3}$ of radius $2 / A$ provided one identifies $\phi$ and $\psi$ with period $2 \sqrt{2} \pi$. This is consistent with the above comment that the $\mu=1$ solution is just the five-dimensional Schwarzschild solution.
}

$$
\delta_{(x=-1)}=2 \pi\left(1-\sqrt{\frac{1-\mu}{1+\mu}}\right) .
$$

In both cases, the $x \phi$ part of the metric describes a surface that is topologically $S^{2}$ with a conical singularity at one of the poles. In the full metric, this singularity is extended in two other spatial dimensions and hence it describes a "deficit membrane," the five-dimensional analogue of a fourdimensional deficit string. ${ }^{8}$

As $y \rightarrow-1, \quad g_{\psi \psi}$ tends to zero. To analyze this, set $y=$ $-\cosh (\xi / \sqrt{1+\mu})$. Near $\xi=0$, the $y \psi$ part of the metric is conformal to

$$
d s_{y \psi}^{2} \approx d \xi^{2}+\frac{\xi^{2}}{1+\mu} d \psi^{2} .
$$

This is regular at $\xi=0$ provided $\psi$ is identified with period $\Delta \psi=2 \pi \sqrt{1+\mu} . y=-1$ is then seen as the origin of polar coordinates and hence $y$ cannot be continued beyond -1 . Returning to the horizon metric (4.25), it is now clear that the topology of the horizon is $S^{1} \times S^{2}$, which justifies calling this solution a black ring. The circumference of the ring varies from a maximum of $2 \pi(1+\mu) A^{-1}$ at $x=-1$ to a minimum of $2 \pi \sqrt{1-\mu^{2}} A^{-1}$ at $x=1$. Since $x$ is the polar coordinate on the $S^{2}$, it follows that $x=-1$ points away from the ring and $x=+1$ points into the hole in the center of the ring. Thus the choice of where to put the conical deficit corresponds either to having the black ring sitting on the rim of a disk shaped deficit membrane (with negative deficit), or to having the black ring sitting on the rim of a disk-shaped hole in an infinitely extended deficit membrane (with positive deficit). The area of the horizon is

$$
\mathcal{A}_{h}=8 \pi^{2} \frac{\mu^{2}(1+\mu)}{A^{3}}
$$

in the former case, and

$$
\mathcal{A}_{h}=8 \pi^{2} \frac{\mu^{2} \sqrt{1-\mu^{2}}}{A^{3}}
$$

in the latter.

It is clear from the metric that the only values of $x$ and $y$ that can correspond to asymptotic infinity are $x=y=-1$. As these values are approached, the metric takes the asymptotic form

$$
\begin{aligned}
d s^{2} \sim & -d t^{2}+\frac{1}{\widetilde{A}^{2}(x-y)^{2}}\left[\left(y^{2}-1\right) d \widetilde{\psi}^{2}+\frac{d y^{2}}{y^{2}-1}+\frac{d x^{2}}{1-x^{2}}\right. \\
& \left.+\left(1-x^{2}\right) d \widetilde{\phi}^{2}\right],
\end{aligned}
$$

\footnotetext{
${ }^{8}$ For a simpler example of a deficit membrane, consider the metric $d s^{2}=-d t^{2}+d r_{1}^{2}+r_{1}^{2} d \theta_{1}^{2}+d r_{2}^{2}+r_{2}^{2} d \theta_{2}^{2}$ where $\theta_{1}$ is identified with period $2 \pi$ and $\theta_{2}$ with period $2 \pi-\delta$. The deficit membrane sits at $r_{2}=0$.
} 
where $\widetilde{\psi}=\psi / \sqrt{1+\mu}, \widetilde{\phi}=\phi / \sqrt{1+\mu}$, and $\widetilde{A}=A /(1+\mu)$. The quantities $\widetilde{\phi}, \quad \widetilde{\psi}$, both have period $2 \pi$ if the period of $\phi$ is chosen such that the conical deficit lies at $x=1$. This metric is in fact known to be flat space. The transformation

$$
\xi=\frac{\sqrt{y^{2}-1}}{\widetilde{A}(x-y)}, \quad \eta=\frac{\sqrt{1-x^{2}}}{\widetilde{A}(x-y)},
$$

takes it to the form

$$
d s^{2} \sim-d t^{2}+d \xi^{2}+d \eta^{2}+\xi^{2} d \widetilde{\psi}^{2}+\eta^{2} d \widetilde{\phi}^{2},
$$

which is free of conical singularities if $\widetilde{\psi}$ and $\widetilde{\phi}$ both have period $2 \pi$, which they do if the conical deficit lies at $x=1$. If the conical deficit lies at $x=-1$ then $\widetilde{\phi}$ has a conical deficit $\delta_{2}$ given by Eq. (4.29), so the asymptotic metric describes a flat deficit membrane in this case. The structure of the black ring is summarized in Fig. 4.

It has been shown that the black ring has an event horizon of topology $S^{2} \times S^{1}$. Naively, one might expect such a horizon to collapse to form a spherical black hole horizon. However, the solution has conical singularities that prevent this from occurring. These conical singularities describe a deficit membrane that either extends to infinity or forms a disk inside the ring. In the latter case, the solution is asymptotically flat. We believe this to be the first example of an asymptotically flat solution with an event horizon of nonspherical topology. Of course, this solution requires the presence of a conical excess angle, which corresponds to a deficit membrane of negative tension. This is presumably unphysical, but it will be shown in [25] that the conical singularity can be eliminated if the ring rotates in the $\psi$ direction.

If the asymptotic metric does not contain a conical singularity then the mass of the black ring can be calculated by considering the subleading contribution to $g_{t t}$. It is easy to show that near $x=y=-1$ this behaves as

$$
g_{t t} \sim-\left(1-\frac{2 \mu(1+\mu)}{A^{2}\left(\xi^{2}+\eta^{2}\right)}+\cdots\right),
$$

from which it follows (see e.g., [18]) that the black ring has mass

$$
M=\frac{3 \pi \mu(1+\mu)}{4 G_{5} A^{2}}
$$

where $G_{5}$ is Newton's constant in five dimensions. Note that when $\mu=1$ this gives the correct value for a fivedimensional Schwarzschild black hole of horizon radius $2 / A$.

If, on the other hand, the deficit membrane extends to infinity, the mass of the ring can be calculated by taking as a reference background the spacetime of a membrane (without a ring), with the result

$$
M=\frac{3 \pi \mu \sqrt{1-\mu^{2}}}{4 G_{5} A^{2}} .
$$

The temperature of the black ring can be obtained by Euclideanizing the near-horizon metric: $t=-i \tau$. In order to avoid a new conical singularity at $y=-\infty$, it is necessary to periodically identify $\tau$. From Eq. (4.21), one finds that the temperature is

$$
T=\frac{A}{4 \pi \mu} .
$$

The topology of the Euclidean solution is $\left(S^{3} \times S^{2}\right)-S^{1}$, where the $S^{3}$ is covered by the coordinates $\tau, \psi$, and $y$, and the $S^{2}$ by $x$ and $\phi$. The circle removed is the circle at $x=y$ $=-1$ parametrized by $\tau$.

For either choice of the position of the deficit membrane, $x=1$ or $x=-1$, there is a Smarr relation:

$$
M=\frac{3}{8 G_{5}} T \mathcal{A}_{h} .
$$

Let us now assume the deficit membrane is outside the ring. The action of the Euclidean solution can be computed similarly to [24], by subtracting the action of the deficit membrane spacetime, and yields

$$
I=\frac{\pi^{2} \mu^{2} \sqrt{1-\mu^{2}}}{G_{5} A^{3}}=\frac{M}{3 T} .
$$

If we now identify the free energy as $F=T I=M-T S$, then using Eq. (4.40) we find that the entropy satisfies the area law

$$
S=\frac{\mathcal{A}_{h}}{4 G_{5}}=\frac{2 \pi^{2} \mu^{2} \sqrt{1-\mu^{2}}}{G_{5} A^{3}} .
$$

It is unclear whether the black ring is a stable solution, or whether it will become unstable for a certain range of parameter values. When the radius of the $S^{1}$ grows to infinity we recover a translationally invariant black string, which is known to be unstable [31], and this suggests that the instability might set in already for finite but large enough radius. In that case the ring would be unstable to rippling along the $\psi$ direction. Given the presence of the deficit membrane, a detailed analysis is needed to settle the issue.

\section{E. Superposition of a black hole and KK bubble}

All of the metrics discussed above were already known ${ }^{9}$ rather than discovered using the general formalism of Sec. II. However, in this section and the following section, new class II solutions will be constructed by following the steps described there.

The first example is the $D=5$ solution that was labeled II(c) above. It will be convenient to parametrize these sources slightly differently from above, taking $U_{1}$ to be the potential of a finite $\operatorname{rod} \mu /(2 A) \leqslant z \leqslant 1 /(2 A), \quad U_{2}$ to be the potential of a finite $\operatorname{rod}-\mu /(2 A) \leqslant z \leqslant \mu /(2 A)$ and $U_{3}$ to be

\footnotetext{
${ }^{9}$ Although the black ring metric had not been interpreted as such.
} 


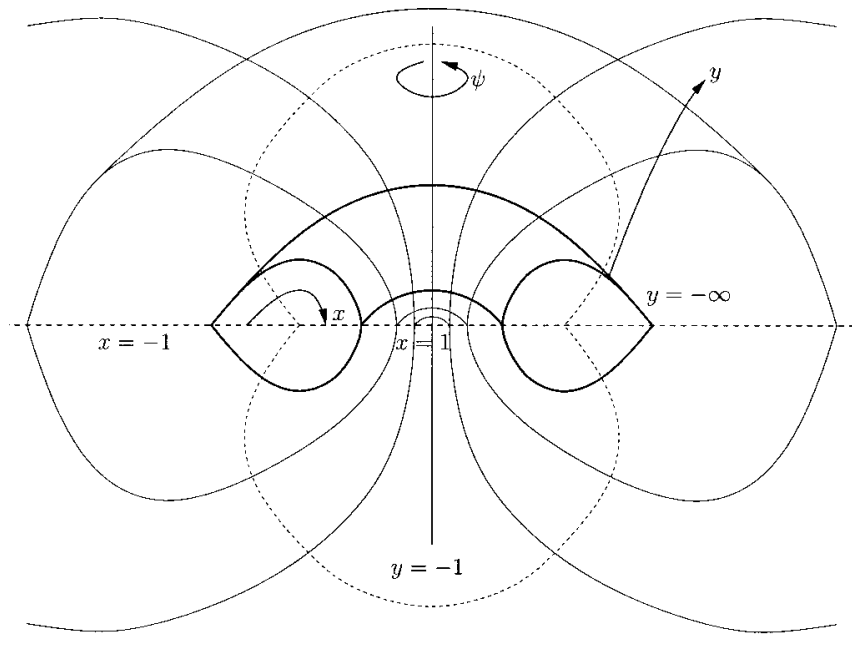

FIG. 4. Spatial sections of the black ring metric. The coordinate $\phi$ is suppressed. The surfaces of constant $y$ are nested surfaces of topology $S^{2} \times S^{1}$. The coordinate $\psi$ is the coordinate on $S^{1}$. The coordinates $x$ and $\phi$ are, respectively, the polar and azimuthal angles on $S^{2}$. The smallest constant $y$ surface corresponds to the horizon, at $y=-\infty$. The surface at $y=-1$ degenerates into an axis of rotation where the orbits of $\psi$ shrink to zero. The surfaces of constant $x$ are denoted by dotted lines. $x=-1$ points out of the ring and $x=+1$ points into the ring. The conical singularity may be chosen to lie inside the ring or, as in the case shown, outside the ring (so that it extends to infinity). Infinity is at $x=y=-1$.

the potential of the semi-infinite rods $z \geqslant 1 /(2 A)$ and $z \leqslant$ $-\mu /(2 A)$. The parameter $\mu$ will be taken in the range 0 $<\mu<1$ (in order to prevent the rods from overlapping). Using the same notation as for the black ring, this gives

$$
\begin{aligned}
e^{2 U_{1}} & =e^{2 u_{1}} \frac{R_{2}-\zeta_{2}}{R_{1}-\zeta_{1}}, \\
e^{2 U_{2}} & =e^{2 u_{2}} \frac{R_{3}-\zeta_{3}}{R_{2}-\zeta_{2}}, \\
e^{2 U_{3}} & =e^{2 u_{3}}\left(R_{1}-\zeta_{1}\right)\left(R_{3}+\zeta_{3}\right),
\end{aligned}
$$

where the $u_{i}$ are arbitrary constants that reflect the freedom to rescale the coordinates $x^{i}$. This will be used to avoid any conical singularities along the axes: according to the general comments of Sec. IV A, they can all be eliminated.

Following the prescription of Sec. II, one now computes the function $\nu$ by writing the functions $U_{i}$ in terms of the complex coordinate $w=r+i z$ and then integrating Eqs. (2.20) and (2.21). This calculation is performed in Appendix E. The result is

$$
e^{2 \nu}=\frac{e^{2 \gamma_{0}}}{R_{1} R_{2} R_{3}} \sqrt{Y_{12} Y_{13} Y_{23}} \frac{R_{1}-\zeta_{1}}{R_{3}-\zeta_{3}},
$$

where $\gamma_{0}$ is an arbitrary constant of integration. The quantities $R_{i}, \zeta_{i}$, and $Y_{i j}$ are the same as for the black ring, Eqs. (4.12), (4.13), and (4.14).
For the black ring, the coordinate transformation $(r, z)$ $\rightarrow(x, y)$ [defined by Eqs. (4.6), (4.10) with $\alpha=A]$ gives a form of the metric that is easier to analyze. This suggests performing the same coordinate transformation here. The transformation of $d r^{2}+d z^{2}$ under this change of coordinates can be obtained from the above analysis of the black ring. In these new coordinates, the metric takes the form

$$
\begin{aligned}
d s^{2}= & -\frac{F(x)}{F(y)} d t^{2}+e^{2 u_{1}} \frac{(1-x) F(y)}{(1-y) F(x)}\left(d x^{1}\right)^{2}+\frac{e^{2 u_{3}}}{A^{4}} \\
& \times \frac{(1+x)(1-y)^{2}(-1-y) F(x) F(y)}{(x-y)^{4}}\left(d x^{3}\right)^{2} \\
& +\frac{\sqrt{2}(1+\mu) e^{2 \gamma_{0}}}{A^{4}(x-y)^{3}}\left[\frac{(1-y) F(x)}{-1-y} d y^{2}\right. \\
& \left.+\frac{(1-y)^{2} F(y)}{1-x^{2}} d x^{2}\right]
\end{aligned}
$$

where the similarity with the black ring has suggested taking $x^{2}$ to be the time direction, normalized such that $u_{2}=0$. The square of the Riemann tensor diverges at $x=1 / \mu$, at $y$ $=1 / \mu$ and at $y=1$. This suggests taking the ranges of the coordinates to be, again, $-1 \leqslant x \leqslant 1$ and $y \leqslant-1$.

This metric has an event horizon at $y=-\infty$, just as for the black ring. The orbits of $x^{1}$ shrink to zero size at $x=1$. Regularity requires that $x^{1}$ is identified with period $2 \pi$ and

$$
e^{2 u_{1}}=2 \sqrt{2}\left(1-\mu^{2}\right) A^{-4} e^{2 \gamma_{0}} .
$$

The orbits of $x^{3}$ shrink to zero size at both $x=-1$ and $y=$ -1 . The metric will be regular in both cases if $x^{3}$ is identified with period $2 \pi$ and

$$
e^{2 u_{3}}=2 \sqrt{2} e^{2 \gamma_{0}} .
$$

Having made these identifications, the above metric is complete and nonsingular and cannot be extended except through the event horizon at $y=-\infty$. Note that $\gamma_{0}$ could be absorbed into $A$ and can therefore be chosen to take any convenient value. The choice

$$
e^{2 \gamma_{0}}=\frac{A^{2}}{\sqrt{2}(1+\mu)}
$$

will be made here. Letting $\phi=x^{1}$ and $\psi=x^{3}$, the metric now takes the form

$$
\begin{aligned}
d s^{2}= & -\frac{F(x)}{F(y)} d t^{2}+\frac{2(1-\mu)}{A^{2}} \frac{(1-x) F(y)}{(1-y) F(x)} d \phi^{2} \\
& +\frac{2(1+x)(1-y)^{2}(-1-y) F(x) F(y)}{(1+\mu) A^{2}(x-y)^{4}} d \psi^{2} \\
& +\frac{1}{A^{2}(x-y)^{3}}\left[\frac{(1-y) F(x)}{-1-y} d y^{2}+\frac{(1-y)^{2} F(y)}{1-x^{2}} d x^{2}\right],
\end{aligned}
$$


where $\phi$ and $\psi$ both have period $2 \pi$. The event horizon at $y=-\infty$ has topology $S^{3}$.

To interpret this metric, it is helpful to look first at certain limiting cases. If $\mu \rightarrow 0$ then the sources for this solution tend to the sources for a metric consisting of a flat time direction times the $D=4$ Euclidean Schwarzschild solution. This is the metric of a static KK bubble. If $\mu \rightarrow 1$ then the sources tend to the sources for a $D=5$ black string, with $\phi$ becoming the translation coordinate along the string (one has to rescale $\phi$ by $\sqrt{1-\mu}$ before taking $\mu \rightarrow 1$ ). Hence this metric must somehow interpolate between a static KK bubble and a black string.

Asymptotic infinity is at $x=y=-1$. Near $x=y=-1$, the metric takes the form ${ }^{10}$

$$
\begin{aligned}
d s^{2} \sim & -d t^{2}+\frac{2}{\widetilde{A}^{2}} \frac{(1-x)}{(1-y)} d \widetilde{\phi}^{2} \\
& +\frac{2(1+x)(1-y)^{2}(-1-y)}{\widetilde{A}^{2}(x-y)^{4}} d \psi^{2} \\
& +\frac{1}{\widetilde{A}^{2}(x-y)^{3}}\left[\frac{(1-y) F(x)}{-1-y} d y^{2}+\frac{(1-y)^{2} F(y)}{1-x^{2}} d x^{2}\right],
\end{aligned}
$$

where $\tilde{A}=A / \sqrt{1+\mu}$ and $\widetilde{\phi}=\sqrt{(1-\mu) /(1+\mu)} \phi$. This metric is what one would obtain from the full metric (4.51) with parameters $(\tilde{\mu}, \tilde{A})$, where $\tilde{\mu}=0$. It must therefore be the metric of a static KK bubble. The periodicity of $\widetilde{\phi}$ is inconsistent with regularity at $x=1$ but this metric is only supposed to be an approximation to the metric (4.51) near $x=y=-1$. The important point is that the static KK bubble is known to be asymptotic to $R^{1,4} \times S^{1}$, which is the KK vacuum metric. It follows that the metric (4.51) must also be asymptotic to the KK vacuum, with the KK circle parametrized by $\phi$.

The orbits of $\psi$ shrink to zero size at $x=-1$ and at $y=$ -1 . To understand what this means, it is convenient to consider the KK bubble $(\mu=0)$ metric and how the coordinates $(x, y)$ relate to the Schwarzschild coordinates $(R, \theta)$ in this case. This can be done by setting $g_{\psi \psi}$ equal to ${ }^{11} 1-2 M / R$ and $g_{\phi \phi}$ equal to $R^{2} \sin ^{2} \theta$. Then $x=-1$ corresponds to the axis $\theta=0$ and $y=-1$ to the axis $\theta=\pi$. The surfaces of constant $x$ and $y$ take the form shown on the left in Fig. 5 .

The metric (4.51) contains a horizon at $y=-\infty$. At that point, the radius of the KK circle is finite. This leads to the picture on the right in Fig. 5. The full geometry of the spatial sections can be visualized by considering how the KK di-

\footnotetext{
${ }^{10}$ Near $x=y=-1$ one has $1-x \approx 2$ etc.; however factors of 1 $-x$ etc. have been retained here for purposes of comparison with the KK bubble.

${ }^{11}$ The value of $M$ can be fixed by looking at the sources in the Weyl form of the metric and comparing with the Weyl form of the Schwarzschild metric: the lengths of the rods should match.
}

mension varies. This is depicted in Fig. 6. The solution describes a black hole sitting in the "throat" of a static KK bubble.

In order to see that the topology of the horizon is $S^{3}$, note first that the structure of the sources around the rod for the time coordinate $x^{2}$ in Fig. 3(c) is locally the same as the rod structure in Fig. 2(b) (with $x^{1}$ as time). In more detail, note that for the KK bubble, the KK circle closes off at $r=2 M$ on a $S^{2}$. Let $\theta \in[0, \pi]$ denote the polar coordinate on this sphere. If the black hole is now included then its horizon intersects the $S^{2}$ at a circle (parametrized by $\psi$ ) at, say, $\theta$ $=\theta_{*}$ with the exterior region at $0 \leqslant \theta<\theta_{*}$. As one moves out of the throat, the $S^{2}$ expands, the KK circle opens up and $\theta_{*}$ increases. Eventually, $\theta_{*}$ reaches $\pi$ and the horizon is no longer present. One can choose coordinates on the horizon to be $\theta_{*}, \phi$, and $\psi$. At the initial value of $\theta_{*}$, the circle parametrized by $\phi$ shrinks to zero, and at the final value $\theta_{*}$ $=\pi$, the circle parametrized by $\psi$ shrinks to zero, from which it follows that the topology of the horizon is $S^{3}$. If $\mu \rightarrow 0$ then the black hole horizon shrinks to zero, leaving a KK bubble. If $\mu \rightarrow 1$ then the horizon grows until it swallows the throat of the bubble. When this happens, the KK direction no longer closes off, and one is left with a compactified black string with an event horizon of topology $S^{2} \times S^{1}$.

If one Euclideanizes the solution then conical singularities can be avoided if the Euclidean time direction $\tau$ is periodically identified with a period $\beta=1 / T$, corresponding to a temperature

$$
T=\frac{A}{4 \pi \sqrt{\mu}} .
$$

This instanton can probably be interpreted as describing an instability of flat space at finite temperature in KK theory. This is a simultaneous manifestation of two different instabilities: the bubble nucleation instability of the KK vacuum [19] and the black hole nucleation instability of flat space at finite temperature [23]. The instanton might also be used to describe a decay of a compactified black string by KK bubble nucleation. Presumably this instanton is not allowed when fermions are included.

It is known that the static KK bubble is classically unstable so it seems likely that a similar instability will afflict this solution. One might therefore wonder whether there is an analogue of the expanding KK bubble solution (described by the Wick rotated $D=5$ Schwarzschild solution [19]) describing a black hole sitting in the throat of the expanding bubble. Such a solution does indeed exist, and is obtained by a Wick rotation of the black ring solution: if one lets $t \rightarrow$ $-i \tau$ and $\phi \rightarrow i t$ in the black ring solution then one obtains the metric ${ }^{12}$

\footnotetext{
${ }^{12}$ In terms of the original notation for Fig. 3(b), this means that the time coordinate is $x^{3}$.
} 


$$
\begin{aligned}
d s^{2}= & \frac{F(x)}{F(y)} d \tau^{2}+\frac{1}{A^{2}(x-y)^{2}}\left[F ( x ) \left(\left(y^{2}-1\right) d \psi^{2}\right.\right. \\
& \left.\left.+\frac{F(y)}{y^{2}-1} d y^{2}\right)+F(y)^{2}\left(\frac{d x^{2}}{1-x^{2}}-\frac{1-x^{2}}{F(x)} d t^{2}\right)\right] .
\end{aligned}
$$

The causal structure of this metric can be understood by first examining the $x t$ part:

$$
d s^{2}=\frac{d x^{2}}{1-x^{2}}-\frac{1-x^{2}}{F(x)} d t^{2}
$$

By changing to Kruskal coordinates, it can be seen that there are regular horizons at $x= \pm 1$ with different surface gravities. The coordinates $(x, t)$ can be reintroduced beyond these horizons. Continuing beyond the horizon at $x=1$, one finds that the square of the Riemann tensor diverges at $x=1 / \mu$. Beyond the horizon at $x=-1$, the metric is asymptotically de Sitter. Figure 7 shows the Carter-Penrose diagram for this two-dimensional metric. The causal structure is the same as Schwarzschild-de Sitter with the horizon at $x=1$ corresponding to the black hole horizon and the horizon at $x=$ -1 corresponding to the cosmological horizon. As for Schwarzschild-de Sitter it appears that there are many black holes and asymptotic regions present, but one is free to identify these if one chooses. Doing so clearly makes the spatial sections compact.

It is easy to see that the full five-dimensional metric will also have regular horizons at $x= \pm 1$. The horizon at $x=1$ has topology $S^{3}$ and the one at $x=-1$ has topology $S^{1}$ $\times R^{2}$ (with the $S^{1}$ direction parametrized by $\psi$ ). Continuing beyond the horizon at $x=1$, there is a curvature singularity as above. Asymptotic infinity is at $x=y$ and lies beyond the horizon at $x=-1$. Near infinity, the metric approaches the KK vacuum with the KK circle parametrized by $\tau$. The causal structure is illustrated in Fig. $7 .{ }^{13}$ The interpretation of this metric is that the horizon at $x=1$ is a black hole horizon (or horizons) and the horizon at $x=-1$ an acceleration horizon that separates causal curves that can fall into the black hole from those that cannot owing to the expansion of space between them and the hole. This expansion of space is just the expansion of the throat region of a $\mathrm{KK}$ bubble, which is where the black hole is located. The asymptotic region beyond the acceleration horizon is the region outside the bubble.

\section{F. Superposition of a black string and KK bubble}

We parametrize the sources for the type II(d) solution as follows. $U_{1}$ is the potential of a semi-infinite $\operatorname{rod} z$ $\geqslant 1 /(2 A), \quad U_{2}$ is the potential of a semi-infinite rod $z \leqslant$ $-\mu /(2 A), \quad U_{3}$ is the potential of a finite $\operatorname{rod}-\mu /(2 A)$

\footnotetext{
${ }^{13} \mathrm{Null}$ infinity is presumably incomplete, as for the expanding KK bubble [20].
}
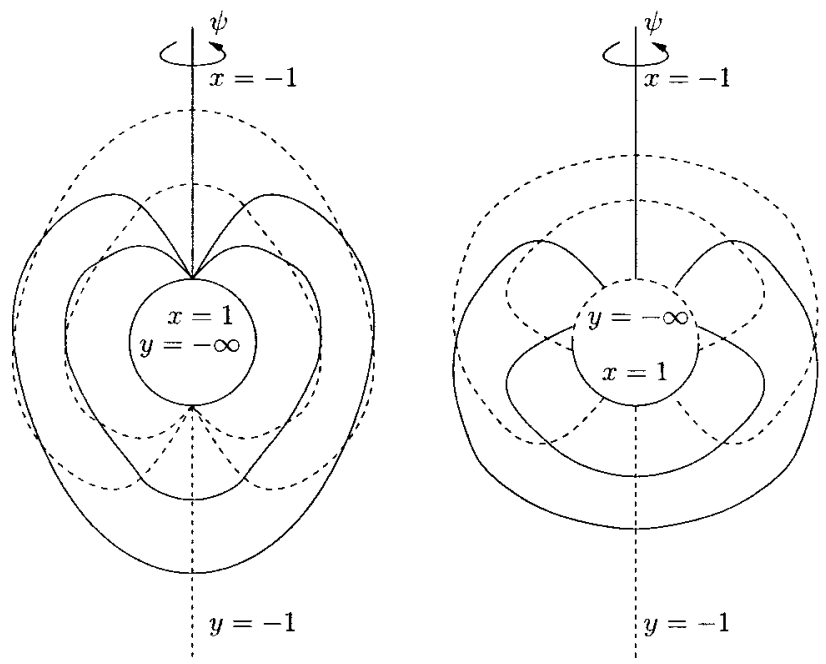

FIG. 5. (1) Schematic depiction of the $R \theta$ plane of the KK bubble. The throat of the bubble (where the KK circle shrinks to zero size) is at $R=2 M$. In the $(x, y)$ coordinates, this corresponds to $x=1$ or $y=-\infty$. The axes $\theta=0, \pi$ correspond to $x=-1$ and $y=$ -1 respectively. Solid and dashed lines denote curves of constant $x$ and $y$ respectively. (2) The $x y$ plane of the metric (4.51). There is a horizon at $y=-\infty$ and the KK circle shrinks to zero size at $x=1$.

$\leqslant z \leqslant \mu /(2 A)$ and $U_{4}$ is the potential of a finite $\operatorname{rod} \mu /(2 A)$ $\leqslant z \leqslant 1 /(2 A)$. This gives

$$
\begin{aligned}
e^{2 U_{1}} & =e^{2 u_{1}}\left(R_{1}-\zeta_{1}\right), \\
e^{2 U_{2}} & =e^{2 u_{2}}\left(R_{3}+\zeta_{3}\right), \\
e^{2 U_{3}} & =e^{2 u_{3}} \frac{R_{3}-\zeta_{3}}{R_{2}-\zeta_{2}},
\end{aligned}
$$

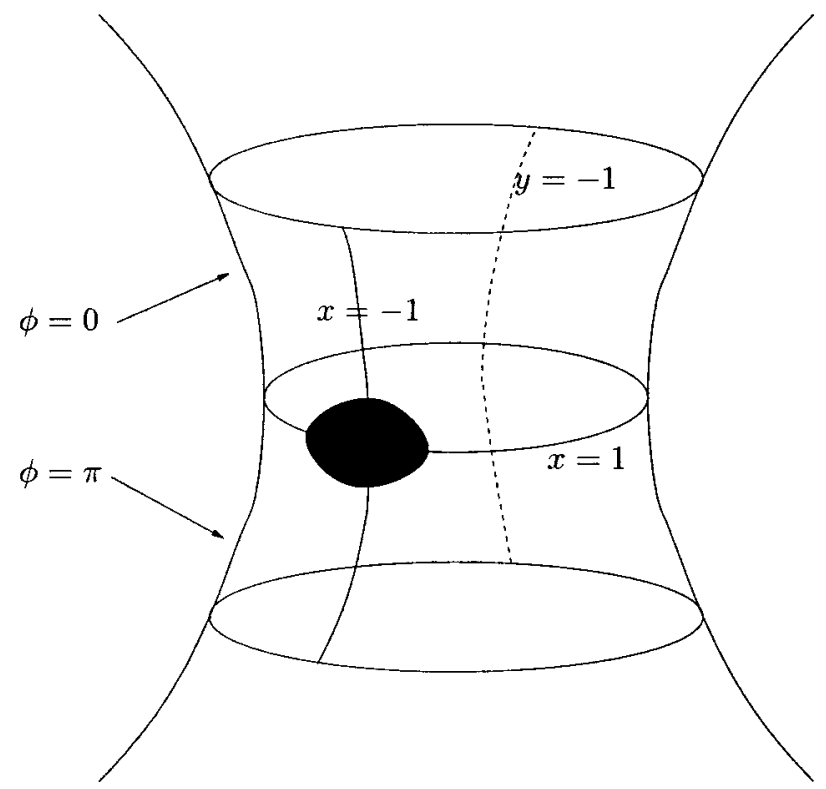

FIG. 6. Geometry of the metric (4.51). This picture shows the surfaces $\phi=0$ (upper half) and $\phi=\pi$ (lower half), which join together smoothly at $x=1$. The horizon at $y=-\infty$ corresponds to a black hole sitting at the center of the "throat" of a static KaluzaKlein bubble. 


$$
e^{2 U_{4}}=e^{2 u_{4}} \frac{R_{2}-\zeta_{2}}{R_{1}-\zeta_{1}},
$$

where the $u_{i}$ are arbitrary constants. As in the previous configuration, all conical singularities can be cancelled by an appropriate choice of these constants and periodic identifications.

The function $\nu$ is calculated using the method of Appendix E with the result

$$
e^{2 \nu}=\frac{e^{2 \gamma_{0}}}{R_{1} R_{2} R_{3}} \sqrt{Y_{12} Y_{23}} \sqrt{\frac{R_{1}-\zeta_{1}}{R_{3}-\zeta_{3}}},
$$

where $\gamma_{0}$ is arbitrary. Once again, it proves useful to convert from the Weyl coordinates $(r, z)$ to the black ring coordinates $(x, y)$ using Eqs. (4.6) and (4.10) (with $\alpha=A$ ). This leads to

$$
\begin{aligned}
d s^{2}= & -\frac{F(x)}{F(y)} d t^{2}+e^{2 u_{4}} \frac{(1-x) F(y)}{(1-y) F(x)}\left(d x^{4}\right)^{2} \\
& +\frac{2 e^{2 \gamma_{0}}}{A^{2}(x-y)^{2}}\left\{F ( x ) \left[\frac{d y^{2}}{-1-y}+\frac{1}{2} e^{2\left(u_{1}-\gamma_{0}\right)}\left(y^{2}-1\right)\right.\right. \\
& \left.\times\left(d x^{1}\right)^{2}\right]+(1-y) F(y)\left[\frac{d x^{2}}{1-x^{2}}+\frac{1}{2} e^{2\left(u_{2}-\gamma_{0}\right)}(1+x)\right. \\
& \left.\left.\times\left(d x^{2}\right)^{2}\right]\right\} .
\end{aligned}
$$

The coordinate $x^{3}$ has been chosen as the time coordinate and normalized so that $u_{3}=0$. The ranges of the coordinates will again be taken to be $-1 \leqslant x \leqslant 1$ and $y \leqslant-1$.

This metric has an event horizon at $y=-\infty$. The orbits of $x^{2}$ shrink to zero at $x=-1$. The metric will be regular there if $x^{2}$ is identified with period $2 \pi$ and

$$
e^{2 u_{2}}=4 e^{2 \gamma_{0}}
$$

The orbits of $x^{4}$ shrink to zero at $x=1$. The metric will be regular there if $x^{4}$ is identified with period $2 \pi$ and

$$
e^{2 u_{4}}=4(1-\mu) A^{-2} e^{2 \gamma_{0}} .
$$

The orbits of $x^{1}$ shrink to zero at $y=-1$. The metric will be regular there if $x^{1}$ is identified with period $2 \pi$ and

$$
e^{2 u_{1}}=4 e^{2 \gamma_{0}}
$$

The constant $\gamma_{0}$ could be absorbed into $A$ and can be conveniently chosen as

$$
e^{2 \gamma_{0}}=\frac{1}{2}
$$

The metric therefore takes the form
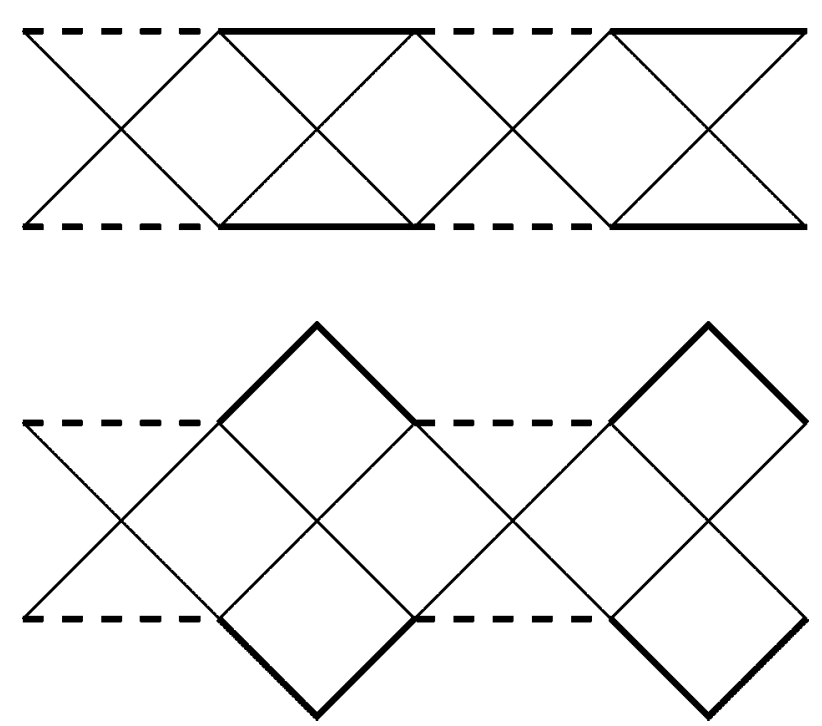

FIG. 7. (1) Causal structure of the two-dimensional metric (4.55). The dotted lines denote curvature singularities, the thick solid lines denote asymptotic infinity and the thin solid lines denote horizons. The pattern can repeat indefinitely to the left and right, or can be made finite by identifications. (2) Causal structure of the Wick rotated black ring metric.

$$
\begin{aligned}
d s^{2}= & -\frac{F(x)}{F(y)} d t^{2}+\frac{2(1-\mu)}{A^{2}} \frac{(1-x) F(y)}{(1-y) F(x)} d \chi^{2} \\
& +\frac{1}{A^{2}(x-y)^{2}}\left\{F(x)\left[\frac{d y^{2}}{-1-y}+2\left(y^{2}-1\right) d \psi^{2}\right]\right. \\
& \left.+(1-y) F(y)\left[\frac{d x^{2}}{1-x^{2}}+2(1+x) d \phi^{2}\right]\right\},
\end{aligned}
$$

where $\chi=x^{4}, \psi=x^{1}$, and $\phi=x^{2}$ all have period $2 \pi$. This metric is complete and nonsingular outside of an event horizon at $y=-\infty$ with topology $S^{3} \times S^{1}$, where the $S^{1}$ is parametrized by $\psi$.

This metric can be analyzed using arguments similar to those of the previous section. It can be seen that the metric is asymptotic to a $D=6$ static KK bubble described by the product of a flat time direction with the $D=5$ Euclidean Schwarzschild solution. It follows that the metric is asymptotic to the $D=6 \mathrm{KK}$ vacuum $M^{1,4} \times S^{1}$, with the $S^{1}$ parametrized by $\chi$. Note that the $S^{1}$ of the horizon does not wrap the KK circle: spacelike infinity and the horizon both have topology $S^{3} \times S^{1}$ but in the former case, the $S^{1}$ is parametrized by $\chi$ and in the latter by $\psi$.

Consider the spatial topology of the static KK bubble. The center of the bubble (where the KK direction collapses) has topology $S^{3}$. Moving out of the bubble, the $S^{3}$ grows and the KK direction opens up so surfaces of constant radius from the bubble have topology $S^{3} \times S_{\chi}^{1}$. For the above solution, this geometry is altered by the presence of an event horizon. This event horizon intersects the minimal $S^{3}$ of the static KK bubble on a $T^{2}$. To see how this happens, introduce coordinates $(\theta, \phi, \psi)$ on the $S^{3}$ such that $\phi$ and $\psi$ correspond to the 
coordinates used above, $0 \leqslant \theta \leqslant \pi / 2$ and the orbits of $\psi$ and $\phi$ collapse at $\theta=0$ and $\theta=\pi / 2$ respectively. For example, the round metric on $S^{3}$ would take the form $d s^{2}=d \theta^{2}$ $+\sin ^{2} \theta d \psi^{2}+\cos ^{2} \theta d \phi^{2}$. The event horizon intersects the minimal $S^{3}$ at some value $\theta=\theta_{*}$, so this intersection has topology $T^{2}=S_{\psi}^{1} \times S_{\phi}^{1}$. The metric outside the event horizon is at $0 \leqslant \theta<\theta_{*}$.

Moving away from the center of the bubble, the $S^{3}$ expands, the KK circle $S_{\chi}^{1}$ opens up and $\theta_{*}$ increases. When $\theta_{*}$ reaches $\pi / 2, \quad S_{\phi}^{1}$ collapses to zero size. Beyond this point, the event horizon no longer intersects the $S^{3}$. The event horizon can therefore be parametrized by the coordinates $\left(\theta_{*}, \chi, \psi, \phi\right)$. At the initial value of $\theta_{*}, \quad S_{\chi}^{1}$ shrinks to a point and at the final value $\theta_{*}=\pi / 2, \quad S_{\phi}^{1}$ shrinks to a point. $S_{\psi}^{1}$ remains finite over the horizon. Hence the horizon has topology $S^{3} \times S_{\psi}^{1}$, where the $S^{3}$ is parametrized by $\left(\theta_{*}, \psi, \phi\right)$.

In the limit $\mu \rightarrow 0$, the event horizon shrinks to nothing and the metric reduces to the static KK bubble. As $\mu \rightarrow 1$, the event horizon grows to engulf the minimal $S^{3}$ and hence there is nowhere that the KK direction collapses. In this limit, the metric reduces to a black string wrapped around the KK direction, so the event horizon has topology $S^{3} \times S_{\chi}^{1}$.

If the metric is Euclideanized by setting $t=-i \tau$ then conical singularities can be avoided by identifying $\tau$ with period $\beta$, corresponding to a temperature

$$
T=\frac{A}{4 \pi \sqrt{\mu}} .
$$

The topology of the Euclidean solution is $\left(S^{3} \times S^{3}\right)-T^{2}$, where one $S^{3}$ is covered by the coordinates $(\tau, \psi, y)$ and the other by $(\chi, \phi, x)$ and the $T^{2}=S_{\chi}^{1} \times S_{\phi}^{1}$ is at $x=y=-1$.

The static KK bubble is known to be unstable, which suggests that this new solution is probably also unstable. For the black-hole bubble solution discussed above, it was possible to obtain the solution describing the evolution of the instability by Wick rotating the black ring. In the present case, however, one can argue that such a solution, if it exists, is not a class II solution, and perhaps not even a Weyl solution.

\section{G. Other Wick rotations}

In order to complete the discussion of these new solutions, this section will discuss the metrics obtained by Wick rotation. Thinking about the sources for the Weyl solutions is useful in understanding what happens when one Wick rotates the class II solutions. For example, in the $C$ metric, $U_{1}$ and $U_{2}$ both have a finite rod and a semi-infinite rod as sources. Therefore it does not matter whether $x^{1}$ or $x^{2}$ is taken to be the time coordinate. For the black ring, the sources are qualitatively different for each $U_{i}$ and hence the choice of which $x^{i}$ is to be the time coordinate leads to physically distinct results. These have all been discussed already. Taking $x^{1}$ to be the time coordinate leads to the black ring. Taking $x^{2}$ to be the time coordinate leads to the solution describing a pair of KK bubbles being accelerated apart by a conical deficit
[24]. Finally, taking $x^{3}$ to be the time coordinate leads to the solution describing an expanding KK bubble with a black hole sitting in the throat.

For the new $D=5$ solution, II(c) [Fig. 3(c)], it is clear that taking $x^{1}$ or $x^{2}$ as the time coordinate gives physically equivalent choices. Above we took $x^{2}$ as time, giving the static black hole-KK bubble metric. However, taking $x^{3}$ to be the time direction leads to a new metric. This is obtained from the metric (4.51) by Wick rotating $t \rightarrow-i \tau$ and $\psi \rightarrow i t$. The resulting metric is asymptotic to $M^{1,2} \times T^{2}$ and has acceleration horizons at $x=-1$ and $y=-1$. The KK directions are parametrized by $\phi$ and $\tau$. Figure 8 shows the geometry of the spatial sections with the KK directions suppressed. The figure on the left shows the region covered by the coordinates $(x, y)$. When the metric is analytically continued beyond the acceleration horizon at $x=-1$ it yields a new region isometric to the first. This can then be continued beyond the horizon at $y=-1$ to yield yet a new region. Therefore, there can be infinitely many such regions. By making identifications, the number of regions can be made finite. For example, the figure on the right shows how to identify in order to obtain just two regions. Figure 9 shows the resulting spatial geometry. The solution describes an expanding KK bubble. The KK circles collapse to zero size in different regions of the bubble's throat. These regions intersect in points. The acceleration horizons separate the regions that these points can causally influence as the throat expands. The Euclidean metric is an instanton for this decay of the $M^{1,2} \times T^{2} \mathrm{KK}$ vacuum.

For the new $D=6$ solution, II(d) [Fig. 3(d)], there are only two physically inequivalent choices of the time coordinate. In the discussion in Sec. IV F $x^{3}$ was chosen as the time direction, giving the static black string-KK bubble metric. The remaining possibility is to take, say, $x^{2}$ to be the time coordinate. This corresponds to the Wick rotation $t \rightarrow$ $-i \tau, \quad \phi \rightarrow i t$ of the metric (4.66). The resulting metric is asymptotic to $M^{1,3} \times T^{2}$, and has an acceleration horizon at $x=-1$. The $\mathrm{KK}$ directions are parametrized by $\chi$ and $\tau$, while $\psi$ is an azimuthal angle at infinity. One can continue through the acceleration horizon as described above, leading to an identical region. This solution can be interpreted as an expanding KK bubble. The $\chi$ and $\tau$ circles collapse in different regions of the bubble's throat. In a dimensionally reduced picture, the KK bubble appears as a singularity of topology $S^{2}$, with $\psi$ the azimuthal angle. The $\chi$ circle collapses near the poles of this sphere, and the $\tau$ circle collapses on the rest of the sphere. The acceleration horizon slices through the equator. If this metric is Euclideanized then it gives an instanton for this decay of the $M^{1,3} \times T^{2}$ vacuum.

\section{H. Different KK reductions}

We have seen in Sec. IV A that if a spatial coordinate $x^{i}$ has only finite rod sources, then it is naturally interpreted as a KK compactified direction. Above we have been considering that points are identified along the orbits of the Killing vector $\xi_{(i)}$. However, when there is more than one Killing direction with compact orbits (whether their radius is asymptotically constant or not) it is possible to perform the identi- 


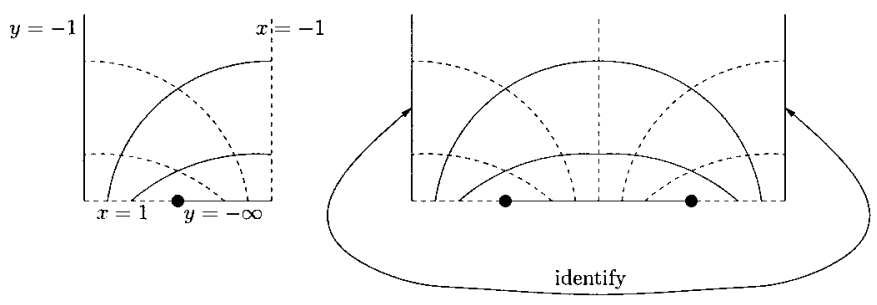

FIG. 8. Spatial sections of the Wick rotated black hole-KK bubble solution (4.51). The KK coordinates $\phi$ and $\tau$ are suppressed. The diagram on the left shows the region covered by the coordinates $(x, y)$. The dotted and solid lines are curves of constant $x$ and $y$ respectively. There are acceleration horizons at $x=-1$ and $y=$ -1 . The $\phi$ direction closes off smoothly at $x=+1$ and the $\tau$ circle closes off smoothly at $y=-\infty$. The heavy dots denote points where both circles close off. The diagram on the right shows how two copies of this region may be pasted together to give a complete geometry.

fications along the orbits of different linear combinations of the Killing vectors. Say that $x^{i}, x^{j}$ are naturally identified with periodicities $\Delta x^{i}, \quad \Delta x^{j}$, in the sense that these identifications result in the absence of (at least some) conical singularities along the axis of symmetry. Then it would also be possible to, instead of identifying points along the orbits of $\xi_{(i)}$, identify them along the orbits of $\xi_{(i)}+\left(\Delta x^{j} / \Delta x^{i}\right) \xi_{(j)}$, with no new singularities arising. If the bolts of $\xi_{(i)}$ and $\xi_{(j)}$ intersect over a common fixed point, then, as shown in [21], the circle action of this linear combination generates a Hopf fibration of $S^{3}$. This change in the global identifications obviously does not affect the local structure of the solution, but it may result in a different interpretation of the dimensionally reduced solution. An exhaustive study of this construction has been performed in [21].

When applied to the Weyl solutions, the most interesting case is that where $x^{i}$ is a KK direction with asymptotically constant radius, and $x^{j}$ is an azimuthal angle. In other words, $U_{i}$ has only finite rod sources, and $U_{j}$ has at least one semiinfinite rod source. In this case, the twisted KK circle action is interpreted as the Hopf fibration of a magnetic monopole. The isolated fixed point of the fibration-the common fixed point of $\xi_{(i)}$ and $\xi_{(j)}$-appears, in the reduced spacetime, as the (singular) source of a magnetic field.

To illustrate this with an example [21], consider adding a flat time direction to the $D=4$ Euclidean Schwarzschild solution [refer to Sec. III B and Fig. 2(a)]. $x^{1}$ is a KK direction, with natural periodicity $8 \pi M$, and $x^{2}$ is an azimuthal angle of period $2 \pi$. With the conventional (untwisted) identifications this describes a static KK bubble. However, $\xi_{(1)}$ $+(1 / 4 M) \xi_{(2)}$ generates Hopf actions with opposite orientations around the end points of the rod. Identifying points along these orbits, these end points appear in the reduced four-dimensional description as a pair of oppositely charged magnetic monopoles. This reduced spacetime is not asymptotically flat: the change in the identifications results also in a KK magnetic Melvin flux tube [12], which balances the attraction between the monopole and the antimonopole. Since the strength of the external magnetic flux tube and the charge of the monopoles are both determined by the amount of twist in the reduction, they are not independent parameters.

Proceeding this way we are led to alternative interpretations of many of the Weyl solutions that contain KK bubbles. The $D=5$ Schwarzschild solution was found to describe in this manner a pair of oppositely charged magnetic monopoles accelerating away under the pull of a magnetic flux tube $[20,21]$. Consider now the solutions in class II(b) [refer to Fig. 3(b)], with $x^{1}$ as the KK coordinate. Dimensionally reduce along the orbits of $\xi_{(1)}+\left(\Delta x^{3} / \Delta x^{1}\right) \xi_{(3)}$, so $x^{2}$ is the timelike coordinate. The end points of the $U_{1}$ rod have the four-dimensional interpretation of a magnetic monopole and antimonopole. $U_{2}$ has a semi-infinite rod, so $x^{2}$ is a boost coordinate and the pair are accelerating, but notice they do it together. By extending the solution across the acceleration horizon, we expect to find a similar monopole-antimonopole dipole accelerating in the opposite direction. The dipoles accelerate under the pull of a magnetic flux tube, and each dipole is held together by the presence of a conical singularity running between the poles.

Now suppose we reduce along the orbits of $\xi_{(1)}$ $+\left(\Delta x^{2} / \Delta x^{1}\right) \xi_{(2)}$. From the sources for the time coordinate $x^{3}$ we infer the presence of an acceleration horizon, but also of a black hole horizon. The vectors $\xi_{(1)}$ and $\xi_{(2)}$ do not share common fixed points: in this case the magnetic charge is not sourced by a monopole, but by a black hole. The interpretation is in terms of a nonextremal KK magnetic black hole moving with uniform acceleration (and its opposite counterpart, from analytic continuation, beyond the acceleration horizon). Since, as we explained, the charge and the magnetic field cannot be varied independently of each other, the solution is only a particular case of the magnetic KK Ernst solution of [12].

In a similar vein, the $\mathrm{II}(\mathrm{c})$ solution with identifications along $\xi_{(2)}+\left(\Delta x^{3} / \Delta x^{2}\right) \xi_{(3)}$ leads to a static configuration with a magnetic monopole and an oppositely charged magnetic black hole. A flux tube keeps them apart in (unstable) equilibrium. The II(d) solution, with an interpretation in

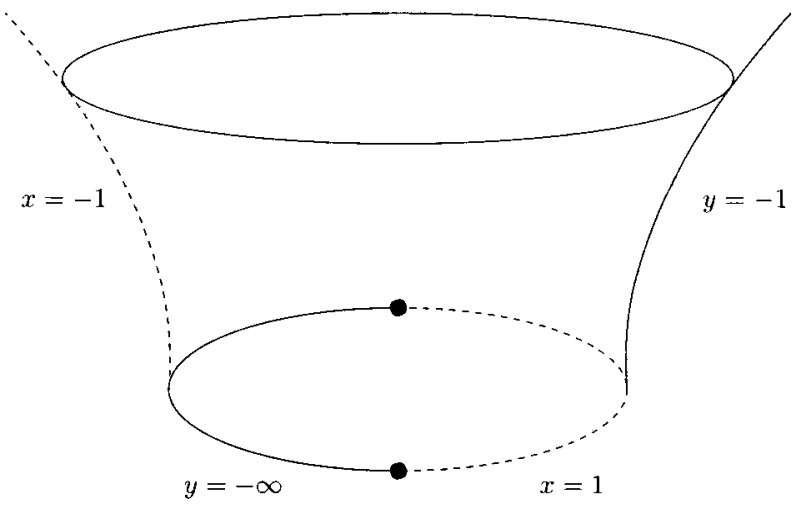

FIG. 9. Throat region of the II(c) solution obtained by Wick rotation of the black hole + bubble metric (4.51) to $t \rightarrow-i \tau, \quad \psi$ $\rightarrow i t$. The $\phi$ and $\tau$ circles close off at $x=+1$ and $y=-\infty$ respectively, denoted by dotted and solid lines. Both circles close off at the points denoted by heavy dots. As the throat expands, the distance between these points increases and the acceleration horizons separate regions which can receive light signals from each point. 
terms of $T^{2}$ compactified KK theory, admits even more combinations, which we shall leave to the reader to analyze.

\section{Multi-black hole configurations}

Weyl's construction in $D=4$ easily allows for configurations with an arbitrary number of black holes along the symmetry axis: these are the Israel-Khan solutions [27]. As is obvious from physical considerations, the conical singularities can only be cancelled if there are an infinite number of them (and then the masses and distances between them are properly adjusted). With such a periodic array of black holes, it is natural to periodically identify the $z$ coordinate, which gives a solution describing a black hole localized on the KK circle of $D=4 \mathrm{KK}$ theory [22].

It is a simple matter to describe Weyl solutions in $D>4$ with several disconnected horizons. This provides the first example of a construction of static multi-black hole solutions in higher dimensional vacuum gravity. Here we will only sketch the properties of the solutions as deduced from their rod structure, rather than giving the full metrics.

The simplest solution with two black hole horizons, each of topology $S^{3}$, results from the class III sources shown in Fig. 10(a). However, due to the isolated finite rod source for $U_{2}$, the coordinate $x^{2}$ is asymptotically a KK circle, so this is not an asymptotically flat solution. Instead, the configuration describes two black holes at the north and south pole of a KK bubble: add a second black hole to Fig. 6, sitting opposite to the one that is already present. The distribution of the sources also reveals that no conical singularities are needed to keep the black holes apart. When the two black holes coalesce, the solution does not reduce to a single, larger, black hole but rather to a black string.

A three-black hole solution is obtained from the sources of Fig. 10(b). This solution is asymptotically flat, with the two spatial Killing directions $x^{2}, \quad x^{3}$ becoming azimuthal angles at infinity. It necessarily contains conical singularities. However, the black holes cannot be described as collinear. The first and second black holes (numbering sources from the left) lie at the north and south pole of a topological $S^{2}$ parametrized by $z$ and $x^{3}$, while the second and third black holes lie similarly on another topological $S^{2}$ parametrized by $z$ and $x^{2}$. So the second black hole is collinear with each of the other two, but along different axes. If two of the black holes coalesce, then we find a configuration of a black hole encircled by a black ring.

Solutions with an infinite number of black hole horizons can be obtained in several ways. The simplest possibility is shown in Fig. 10(c). It is likely that conical singularities can be eliminated from this solution if each $U_{i}$ has sources consisting of rods of equal length. It is natural to periodically identify the $z$ axis to obtain a solution with a single black hole localized on a KK circle, parametrized by $z$, of fixed length at infinity. The coordinates $x^{2}$ and $x^{3}$ also parametrize circles but these do not approach a constant length at infinity so they cannot be regarded as KK directions. Spacelike infinity has topology $S_{2}^{1} \times S_{3}^{1} \times S_{z}^{1}$ rather than $S^{2} \times S_{z}^{1}$ which would be appropriate for a description of a black hole localized on a KK circle. It is clear that other similar configura- tions of infinitely many rods will suffer from the same drawback.

Configurations with multiple concentric black rings are also possible, but we shall stop at this point.

\section{CONCLUDING REMARKS}

We have succeeded in generalizing Weyl's class of solutions to arbitrary dimension by finding the general solution of the vacuum Einstein equations in $D$ dimensions that admits $D-2$ orthogonal commuting non-null Killing vector fields. There are two classes of static Weyl solutions. The first (the "generic" Weyl solutions) is parametrized by $D$ -3 independent axisymmetric harmonic functions in three dimensional flat space. The second class (the "special" Weyl solutions), analyzed in Appendix B, is parametrized by $D$ -4 independent harmonic functions in two dimensional flat space. All known physically relevant solutions fall into the first class, and these solutions were all found to have harmonic functions produced by sources consisting of thin rods on the $z$ axis, with mass $1 / 2$ per unit length. A natural way of classifying the solutions was proposed, according to the number of finite rod sources for the harmonic functions. This classification scheme presented three new solutions as promising candidates for deeper study (see Table I).

Perhaps the most interesting new solution is the black ring of Sec. V C. This is the first example of a stationary solution of the vacuum Einstein equations that is asymptotically flat and has an event horizon of nonspherical topology. ${ }^{14}$ The black ring is supported against collapse by a conical deficit singularity in the form of a disk that sits inside the ring. This singularity might be regarded as the gravitational effect of a thin membrane of matter, much as a deficit string can be regarded as an idealization of a real cosmic string. The deficit for the black ring has negative deficit angle, and hence corresponds to a negative tension source. It is not likely that it can be modeled by any reasonable matter source, since the weak energy condition would not be obeyed. An alternative which is perhaps physically more reasonable is to take the conical deficit to lie outside the ring. A deficit membrane of positive tension is then present which extends to infinity, so the solution is no longer asymptotically flat.

If the black ring were charged, one might envisage balancing it against collapse (and therefore cancelling the conical singularity) by immersing it in a background field. This is actually the situation with the five-dimensional charged black ring solution of [7], which is the first example of a stationary solution with spacelike infinity of spherical topology and a regular horizon of nonspherical topology. The horizon of the ring in that case is an extremal, degenerate one, with vanishing horizon area. It is nevertheless completely

\footnotetext{
${ }^{14} \mathrm{~A}$ toroidal horizon in four dimensions has been observed in numerical simulations of collapse in [32]. It is a transient phase of the collapse: the hole in the torus closes up faster than the speed of light, thereby preventing asymptotic observers from probing the topology of the horizon [33].
} 


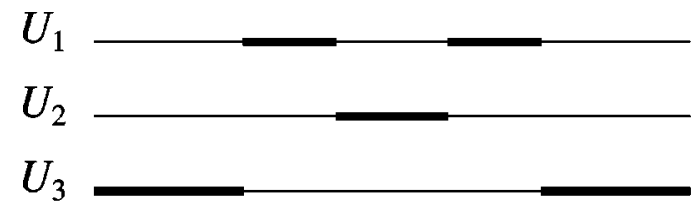

(a)

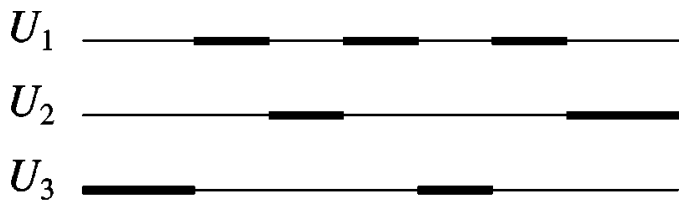

(b)

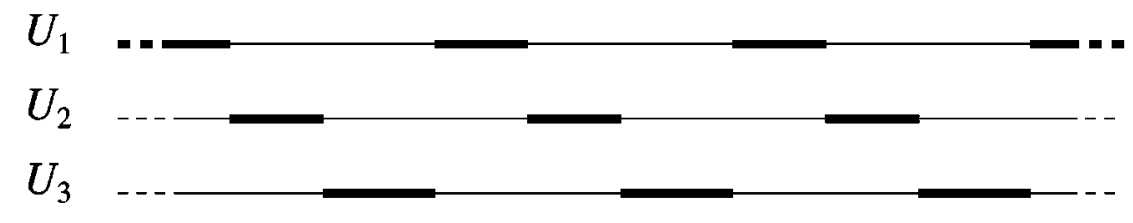

(c)

FIG. 10. Sources for (a) two-black hole configuration, (b) three-black hole configuration, and (c) infinite periodic array of black holes.

nonsingular. The presence of the background field implies that the spacetime is not asymptotically flat, instead it asymptotes to a fluxbrane solution. An alternative to coupling to a background field is to set the ring into rotation. It will be shown in [25] that one can indeed obtain a vacuum solution describing an asymptotically flat rotating black ring ${ }^{15}$ that is free of conical singularities.

The other new solutions contain KK bubbles in addition to black holes or black strings. Some of them describe new decays of the KK vacua with internal $S^{1}$ or $T^{2}$. We have exhibited but a few examples of the wide range of possibilities for solutions which are singular in the KK reduced description but nevertheless completely regular in higher dimensions. Just as the black ring arose from a reinterpretation of a previously known solution, maybe the other class II (or higher) solutions have unexpected applications.

We have sketched the construction of multi-black hole configurations. In contrast to the Israel-Khan solutions in $D$ $=4$, the generalized Weyl solutions cannot describe a linear array of five-dimensional black holes [which would have symmetry $\mathbf{R} \times O(3)$ instead of $\left.\mathbf{R} \times O(2)^{2}\right]$. Nevertheless, we have given the first examples of static vacuum multi-black hole configurations in dimensions higher than four. Unlike the Israel-Khan solutions, some of the higher dimensional solutions with finitely many black holes do not contain conical singularities.

There are several directions for extensions of the Weyl classes in this paper. Consideration of nonorthogonal Killing vectors would lead to nonstatic, stationary solutions, or solutions with twists among the axes. However, in $D=4$ there is no general solution for nonorthogonal Killing vectors, so progress here could probably only be made in special cases. It would also be interesting to study the addition of $p$-form gauge fields.

\footnotetext{
${ }^{15}$ Other recent solutions where rotation plays a role in balancing charged, ring-like or tube-like configurations have been given in [34].
}

To conclude, we have performed a systematic analysis of an infinite class of exact solutions of vacuum gravity in dimensions higher than four, and exhibited new solutions with qualitatively new properties. We hope that this work helps stimulate further systematic studies on the rich structure of exact solutions of higher-dimensional gravity.

\section{ACKNOWLEDGMENTS}

We are grateful to Andrew Chamblin, Fay Dowker, Jerome Gauntlett and Gary Gibbons for discussions. R.E. acknowledges partial support from UPV grant 063.310EB187/98 and CICYT AEN99-0315. H.S.R. was supported by PPARC.

\section{APPENDIX A: CURVATURE COMPONENTS}

We introduce a vielbein for the metric of Eq. (2.3):

$$
e^{i}=e^{U_{i}} d x^{i}, \quad e^{Z}=e^{C} d Z, \quad e^{\bar{Z}}=e^{C} d \bar{Z} .
$$

The summation convention is not being used for the indices $i, j, \ldots$. The tangent space metric $\eta_{\alpha \beta}$ is given by $\eta_{i i}$ $=\epsilon_{i}, \quad \eta_{Z \bar{Z}}=\eta_{\bar{Z} Z}=1 / 2$, with other components vanishing. The connection 1 -forms are defined by

$$
d e^{\alpha}=-\omega_{\beta}^{\alpha} \wedge e^{\beta},
$$

and explicit calculation gives

$$
\begin{gathered}
\omega_{i Z}=e^{-C} \partial_{Z} U_{i} e_{i}, \quad \omega_{i \bar{Z}}=e^{-C} \partial_{\bar{Z}} U_{i} e_{i}, \quad \omega_{i j}=0, \\
\omega_{Z \bar{Z}}=-\frac{1}{2} e^{-C} \partial_{Z} C e^{Z}+\frac{1}{2} e^{-C} \partial_{\bar{Z}} C e^{\bar{Z}} .
\end{gathered}
$$

The curvature 2-forms are defined by

$$
\Theta_{\alpha \beta}=d \omega_{\alpha \beta}+\omega_{\alpha}^{\gamma} \wedge \omega_{\gamma \beta} .
$$

The nonvanishing curvature 2 -forms are 
TABLE I. Summary of the main solutions studied in this paper, in the classification of Sec. IV B. The sources and metrics are referred to the figures and equations in this paper, respectively. Only the topology of finite area horizons is described. Other interpretations, by different Wick rotations or KK reductions, are discussed in Secs. IV G and IV H.

\begin{tabular}{lcccc}
\hline \hline Class & Sources (Fig.) & Metric (Eq.) & Interpretation & Horizon \\
\hline 0 & 1 & $(3.6)$ & Flat (Rindler) space & \\
I & $2(\mathrm{a})$ & 4D Black hole & $S^{2}$ \\
& 2 (b) & $(3.8)$ & 5D Black hole & $S^{3}$ \\
II & $3(\mathrm{a})$ & $C$ metric & $S^{2}$ \\
& $3(\mathrm{~b})$ & $(4.1)$ & Black ring & $S^{2} \times S^{1}$ \\
& $3(\mathrm{~b})$ & $(4.54)$ & Black hole in expanding KK bubble & $S^{3}$ \\
& $3(\mathrm{c})$ & $(4.51)$ & Black hole in static KK bubble & $S^{3}$ \\
& 3(d) & $(4.66)$ & Black string in static KK bubble & $S^{3} \times S^{1}$ \\
& $10(\mathrm{a}, \mathrm{II}, \mathrm{c}, \mathrm{d})$ & & Multi-black holes & $\oplus_{n} S^{3}$ \\
\hline \hline
\end{tabular}

$$
\begin{aligned}
\Theta_{i j}= & -2 e^{-2 C}\left(\partial_{Z} U_{i} \partial_{\bar{Z}} U_{j}+\partial_{Z} U_{j} \partial_{\bar{Z}} U_{i}\right) e_{i} \wedge e_{j}, \\
\Theta_{i Z}= & -e^{-2 C}\left[\partial_{\bar{Z}}^{2} U_{i}+\left(\partial_{Z} U_{i}\right)^{2}-2 \partial_{Z} C \partial_{Z} U_{i}\right] e_{i} \wedge e^{Z} \\
& -e^{-2 C}\left[\partial_{Z} \partial_{\bar{Z}} U_{i}+\partial_{Z} U_{i} \partial_{\bar{Z}} U_{i}\right] e_{i} \wedge e^{\bar{Z}}, \\
\Theta_{i \bar{Z}}= & -e^{-2 C}\left[\partial_{Z} \partial_{\bar{Z}} U_{i}+\partial_{Z} U_{i} \partial_{\bar{Z}} U_{i}\right] e_{i} \wedge e^{Z}-e^{-2 C}\left[\partial_{\bar{Z}}^{2} U_{i}\right. \\
& \left.+\left(\partial_{\bar{Z}} U_{i}\right)^{2}-2 \partial_{\bar{Z}} C \partial_{\bar{Z}} U_{i}\right] e_{i} \wedge e^{\bar{Z}}, \\
\Theta_{Z \bar{Z}}= & e^{-2 C} \partial_{Z} \partial_{\bar{Z}} C e^{Z} \wedge e^{\bar{Z}} .
\end{aligned}
$$

The tangent space components of the Riemann tensor are obtained from these expressions by

$$
\Theta_{\alpha \beta}=\frac{1}{2} R_{\alpha \beta \gamma \delta} e^{\gamma} \wedge e^{\delta},
$$

with the results

$$
R_{i j k l}=-2 e^{-2 C}\left(\partial_{Z} U_{i} \partial_{\bar{Z}} U_{j}+\partial_{Z} U_{j} \partial_{\bar{Z}} U_{i}\right)\left(\eta_{i k} \eta_{j l}-\eta_{i l} \eta_{j k}\right),
$$

$R_{i Z j Z}=-e^{-2 C}\left[\partial_{Z}^{2} U_{i}+\left(\partial_{Z} U_{i}\right)^{2}-2 \partial_{Z} C \partial_{Z} U_{i}\right] \eta_{i j}$

$R_{i \bar{Z} j \bar{Z}}=-e^{-2 C}\left[\partial_{\bar{Z}}^{2} U_{i}+\left(\partial_{\bar{Z}} U_{i}\right)^{2}-2 \partial_{\bar{Z}} C \partial_{\bar{Z}} U_{i}\right] \eta_{i j}$,

$R_{i Z j \bar{Z}}=-e^{-2 C}\left(\partial_{Z} \partial_{\bar{Z}} U_{i}+\partial_{Z} U_{i} \partial_{\bar{Z}} U_{i}\right) \eta_{i j}$,

$R_{Z \bar{Z} Z \bar{Z}}=e^{-2 C} \partial_{Z} \partial_{\bar{Z}} C$,

with any other nonvanishing components related to these by the symmetries of the Riemann tensor. The nonvanishing tangent space components of the Ricci tensor are given by

$$
\begin{aligned}
R_{i j}= & -2 e^{-2 C}\left[2 \partial_{Z} \partial_{\bar{Z}} U_{i}+\partial_{Z} U_{i} \sum_{k} \partial_{\bar{Z}} U_{k}\right. \\
& \left.+\partial_{\bar{Z}} U_{i} \sum_{k} \partial_{Z} U_{k}\right] \eta_{i j}, \\
R_{Z Z}= & -e^{-2 C} \sum_{i}\left(\partial_{Z}^{2} U_{i}+\left(\partial_{Z} U_{i}\right)^{2}-2 \partial_{Z} C \partial_{Z} U_{i}\right), \\
R_{\bar{Z} \bar{Z}}= & -e^{-2 C} \sum_{i}\left(\partial_{\bar{Z}}^{2} U_{i}+\left(\partial_{\bar{Z}} U_{i}\right)^{2}-2 \partial_{\bar{Z}} C \partial_{\bar{Z}} U_{i}\right), \\
R_{Z \bar{Z}}= & -e^{-2 C}\left[2 \partial_{Z} \partial_{\bar{Z}} C+\sum_{i} \partial_{Z} \partial_{\bar{Z}} U_{i}\right. \\
& \left.+\sum_{i} \partial_{Z} U_{i} \partial_{\bar{Z}} U_{i}\right] .
\end{aligned}
$$

\section{APPENDIX B: THE SPECIAL WEYL SOLUTIONS}

When solving the Einstein equations in Sec. II B, a specific assumption was made in order to deal with the $Z Z$ and $\bar{Z} \bar{Z}$ components: that the functions $w(Z)$ and $\tilde{w}(\bar{Z})$ that appear in Eq. (2.6) are nonconstant. In this appendix we investigate the cases in which one or both of these quantities is constant. Consider first the case in which $Z$ and $\bar{Z}$ are complex conjugate coordinates. Then ${ }^{16} \tilde{w}(\bar{Z})=w(Z)^{*}$ so both $w$ and $\tilde{w}$ must be constant. The $i j$ components of the vacuum Einstein equations therefore reduce to

$$
\sum_{i} U_{i}=\text { const }
$$




$$
\partial_{Z} \partial_{\bar{Z}} U_{i}=0
$$

These equations have the solution

$$
U_{i}(Z, \bar{Z})=a_{i}(Z)+a_{i}(Z)^{*}
$$

with $a_{i}(Z)$ arbitrary except for the constraint

$$
\sum_{i} a_{i}(Z)=\text { const. }
$$

The $Z Z$ and $\bar{Z} \bar{Z}$ components of the Einstein equation reduce to

$$
\sum_{i}\left(\partial_{Z} a_{i}\right)^{2}=0
$$

The $Z \bar{Z}$ component of the Einstein equation can be written

$$
\partial_{Z} \partial_{\bar{Z}}\left(C+\frac{1}{4} \sum_{i} U_{i}^{2}\right)=0
$$

with solution

$$
C(Z, \bar{Z})=-\frac{1}{4} \sum_{i} U_{i}^{2}+c(Z)+c(Z)^{*},
$$

where $c(Z)$ is arbitrary. This arbitrary function just reflects the freedom to change coordinates $Z \rightarrow Z^{\prime}(Z)$. Thus the distinct solutions are labeled by the functions $a_{i}(Z)$. These $D$ -2 functions are constrained by Eqs. (B4) and (B5), so only $D-4$ of them are independent. For $D=4$ the solution is flat space. For $D>4$ the solutions are nontrivial and have no four-dimensional analogue. Note that each $U_{i}$ is a solution of the Laplace equation in two-dimensional flat space, so this special class of solutions is determined by $D-4$ harmonic functions in two flat dimensions, in contrast to the class of generic Weyl solutions discussed in Sec. II B, which was determined in terms of $D-3$ axisymmetric harmonic functions in three flat dimensions. In $D=5$, it is possible to explicitly solve the constraints on the $a_{i}$ 's to obtain a line element parametrized by an arbitrary function of one complex coordinate.

Now consider the case in which $Z$ and $\bar{Z}$ are independent real coordinates and $\tilde{w}$ is constant but $w(Z)$ is not. $\tilde{w}$ can be absorbed into $w(Z)$. The $i j$ Einstein equations reduce to

$$
\begin{gathered}
\sum_{i} U_{i}=\log (w(Z)), \\
2 w \partial_{Z} \partial_{\bar{Z}} U_{i}+\partial_{Z} w \partial_{\bar{Z}} U_{i}=0,
\end{gathered}
$$

with solution

$$
U_{i}(Z, \bar{Z})=a_{i}(Z)+\tilde{a}_{i}(\bar{Z}) w^{-1 / 2},
$$

where $a_{i}(Z)$ and $\tilde{a}_{i}(\bar{Z})$ are arbitrary except for the constraints

$$
\sum_{i} \tilde{a}_{i}(\bar{Z})=\tilde{a}
$$

$$
\sum_{i} a_{i}(Z)=\log w-\tilde{a} w^{-1 / 2}
$$

where $\tilde{a}$ is a constant. The $\bar{Z} \bar{Z}$ component of the Einstein equation reduces to

$$
\sum_{i}\left(\partial_{\bar{Z}} \tilde{a}_{i}\right)^{2}=0
$$

The $Z Z$ component of the Einstein equation gives

$$
C(Z, \bar{Z})=\frac{1}{2} \log \partial_{Z} w+\nu+\tilde{c}(\bar{Z}),
$$

where $\tilde{c}(\bar{Z})$ is arbitrary and

$$
\partial_{Z} \nu=-\frac{w}{\partial_{Z} w} \sum_{i<j} \partial U_{i} \partial U_{j}
$$

The $Z \bar{Z}$ component of the Einstein equation is satisfied as a consequence of these equations. The arbitrary function $\widetilde{c}(\bar{Z})$ reflects the freedom to do a coordinate transformation $\bar{Z}$ $\rightarrow \bar{Z}^{\prime}(\bar{Z})$, and one can also do a coordinate transformation $Z \rightarrow w(Z)$ to eliminate $w(Z)$. Hence these solutions are characterized by $D-2$ functions $a_{i}(Z)$ and $D-2$ functions $\tilde{a}_{i}(\bar{Z})$. However, the constraints (B11) and (B13) imply that only $D-4$ of the functions $\tilde{a}_{i}$ are independent, and the constraint (B12) implies that only $D-3$ of the functions $a_{i}$ are independent. For $D=4$, the solution is given by a single arbitrary function $a_{i}(Z)$ and $\partial / \partial \bar{Z}$ is a null Killing vector field so the solution describes a $p p$-wave spacetime [1]. These solutions admit a Killing spinor [35]. The higher dimensional analogues of these $p p$ waves are the solutions with $\tilde{a}_{i}=0$ for all $i$, and it is straightforward to show that these are the only Weyl solutions that satisfy the integrability conditions for the existence of a Killing spinor.

\section{APPENDIX C: WEYL FORMS OF FLAT SPACE}

The results of Appendix A show that the Riemann tensor of the metric (2.3) vanishes if, and only if,

$$
\begin{aligned}
\partial_{Z} U_{i} \partial_{\bar{Z}} U_{j}+\partial_{Z} U_{j} \partial_{\bar{Z}} U_{i} & =0, \quad i \neq j, \\
\partial_{Z}^{2} U_{i}+\left(\partial_{Z} U_{i}\right)^{2}-2 \partial_{Z} C \partial_{Z} U_{i} & =0, \\
\partial_{\bar{Z}}^{2} U_{i}+\left(\partial_{\bar{Z}} U_{i}\right)^{2}-2 \partial_{\bar{Z}} C \partial_{\bar{Z}} U_{i} & =0, \\
\partial_{Z} \partial_{\bar{Z}} U_{i}+\partial_{Z} U_{i} \partial_{\bar{Z}} U_{i} & =0, \\
\partial_{Z} \partial_{\bar{Z}} C & =0 .
\end{aligned}
$$

Equation (C4) can be immediately solved: 


$$
U_{i}(Z, \bar{Z})=\log \left(a_{i}(Z)+a_{i}(Z)^{*}\right),
$$

where $a_{i}(Z)$ is arbitrary.

It is convenient to choose the labeling of the $U_{i}$ such that $\partial_{Z} a_{i} \neq 0$ for $1 \leqslant i \leqslant r$ and $\partial_{Z} a_{i}=0$ for $i>r$. Equation (C2) is automatically satisfied if $i>r$ or $j>r$. If $i, j \leqslant r$ then this equation implies

$$
\frac{\partial_{Z} a_{i}}{\partial_{Z} a_{j}}=-\frac{\left(\partial_{Z} a_{i}\right)^{*}}{\left(\partial_{Z} a_{j}\right)^{*}}=i \lambda_{i j}
$$

where $\lambda_{i j}$ is a real nonzero constant. If $r>2$ then it follows that

$$
\partial_{Z} a_{3}=i \lambda_{32} \partial_{Z} a_{2}=-\lambda_{32} \lambda_{21} \partial_{Z} a_{1}
$$

However, this contradicts $\partial_{Z} a_{3}=i \lambda_{31} \partial_{Z} a_{1}$. Hence $r=0,1$ or 2 . These three cases will be discussed individually.

If $r=0$ then $a_{i}(Z)$ is a constant for all $i$ and hence $U_{i}$ is constant for all $i$. Equations $(\mathrm{C} 2)$ and $(\mathrm{C} 3)$ are trivially satisfied and Eq. (C5) has the solution

$$
C(Z, \bar{Z})=c(Z)+c(Z)^{*},
$$

where $c(Z)$ is arbitrary. This arbitrary function can be eliminated by a coordinate transformation $Z \rightarrow Z^{\prime}(Z)$. The line element is then obviously flat.

If $r=1$ or 2 then Eqs. (C2) and (C3) are trivially satisfied for $i>r$. For $i \leqslant r$, the solution is

$$
C(Z, \bar{Z})=\frac{1}{2} \log \left(\partial_{Z} a_{i}\right)\left(\partial_{Z} a_{i}\right)^{*}+\text { const },
$$

which also ensures that Eq. (C5) is satisfied. For $r=1$, after changing coordinates from $Z$ to $a_{1}(Z)$, setting $a_{1}=\xi+i \eta$ and rescaling the coordinates to eliminate constants, the line element takes the form

$$
d s^{2}=\epsilon_{1} \xi^{2}\left(d x^{1}\right)^{2}+\sum_{i=2}^{D-2} \epsilon_{i}\left(d x^{i}\right)^{2}+d \xi^{2}+d \eta^{2}
$$

This line element is manifestly flat, with $x^{1}$ an angular coordinate (if $\epsilon_{1}=1$ ) or the boost coordinate in Rindler space (if $\left.\epsilon_{1}=-1\right)$.

For $r=2$, after changing and rescaling the coordinates as above, the line element takes the form

$$
d s^{2}=\epsilon_{1} \xi^{2}\left(d x^{1}\right)^{2}+\epsilon_{2} \eta^{2}\left(d x^{2}\right)^{2}+\sum_{i=3}^{D-2} \epsilon_{i}\left(d x^{i}\right)^{2}+d \xi^{2}+d \eta^{2},
$$

which is manifestly flat, with $x^{1}$ an angular or boost coordinate depending on the sign of $\epsilon_{1}$, and similarly for $x^{2}$.

In order to identify the source terms for Laplace's equation that these flat metrics correspond to, one must first change the coordinate from $Z$ to $w(Z)$ [defined by Eq. (2.18) with $\left.\tilde{w}=w^{*}\right]$. However, this is not possible if $r=0$ because then $w$ is constant. Hence $r=0$ corresponds to the special case analyzed in Sec. II D.
For $r=1, \quad U_{i}$ is constant for $i>1$ so Eq. (2.18) reduces to

$$
U_{1}=\log (w+\bar{w})+\text { const }=\log r+\text { const },
$$

where $w=r+i z$. The constant term can be eliminated by rescaling the coordinate $x^{1} . U_{1}$ is the Newtonian potential produced by an infinite rod lying on the $z$ axis. The rod has vanishing thickness and mass $1 / 2$ per unit length.

For $r=2, \quad U_{i}$ is constant for $i>2 . U_{1}$ and $U_{2}$ are given by Eq. (C6). Recall that $\partial_{Z} a_{2}=i \lambda_{21} \partial_{Z} a_{1}$ hence $a_{2}$ $=\lambda_{21}\left(i a_{1}+c\right)$, where $c$ is a constant. The imaginary part of $c$ does not affect $U_{2}$ so $c$ can be taken to be real. Equation (2.18) then gives

$$
w+w^{*} \propto\left[a_{1}+a_{1}^{*}\right]\left[i\left(a_{1}-a_{1}\right)^{*}+2 c\right],
$$

which can be solved to give $a_{1}$ in terms of $w$, and then express $U_{1}$ and $U_{2}$ in terms of $w$ :

$$
\begin{aligned}
& U_{1}=\log |\operatorname{Re} \sqrt{a \pm i w}|+\text { const, } \\
& U_{2}=\log |\operatorname{Re} \sqrt{-a \bar{\mp} i w}|+\text { const, }
\end{aligned}
$$

where $a$ is an arbitrary real constant.

\section{APPENDIX D: FORMULAS FOR THE BLACK RING}

In order to write the metric of the black ring in Weyl form, it turns out to be convenient to look for constants $c$ and $\beta$ such that

$$
r^{2}+\left(z-c / A^{2}\right)^{2}=\frac{1}{4(x-y)^{2} A^{4}}(\alpha \mu x y-2 c x-2 c y+\beta)^{2} .
$$

A priori, one would not expect such constants to exist. However, it turns out that they do, and are given by

$$
c=\frac{\alpha^{2} \mu}{\alpha \mu+\beta},
$$

and

$$
\beta^{3}+\alpha \mu \beta^{2}-\alpha^{2}\left(\mu^{2}+4\right) \beta-\alpha^{3} \mu\left(\mu^{2}-4\right)=0 .
$$

The solutions are

$$
\begin{aligned}
& \beta=\alpha \mu, \quad c=\alpha / 2, \quad \beta=\alpha(2-\mu), \quad c=\alpha \mu / 2, \\
& \beta=-\alpha(2+\mu), \quad c=-\alpha \mu / 2 .
\end{aligned}
$$

Note that the solutions for $c$ can be written as $A^{2} a_{i}$, where the quantities $a_{i}$ were defined in Sec. IV C. It follows that each function $R_{i}$ defined in Sec. IV C is the square root of the right-hand side of Eq. (D1) for the appropriate value of $c$. Since the right-hand side of this equation is a perfect square, the expressions for $R_{i}$ turn out to be quite simple:

$$
R_{1}=\frac{\alpha(\mu x y-x-y+\mu)}{2 A^{2}(x-y)},
$$




$$
\begin{aligned}
& R_{2}=\frac{\alpha(\mu x y-\mu x-\mu y+2-\mu)}{2 A^{2}(x-y)}, \\
& R_{3}=\frac{\alpha(-\mu x y-\mu x-\mu y+2+\mu)}{2 A^{2}(x-y)} .
\end{aligned}
$$

It is also possible to show

$$
\begin{aligned}
R_{1}+\zeta_{1} & =\frac{\alpha\left(1-x^{2}\right) F(y)}{A^{2}(x-y)^{2}}, \\
R_{1}-\zeta_{1} & =\frac{\alpha\left(y^{2}-1\right) F(x)}{A^{2}(x-y)^{2}}, \\
R_{2}+\zeta_{2} & =\frac{\alpha(1+x)(1-y) F(x)}{A^{2}(x-y)^{2}}, \\
R_{2}-\zeta_{2} & =\frac{\alpha(1-x)(-1-y) F(y)}{A^{2}(x-y)^{2}}, \\
Y_{12}= & \frac{\alpha^{2}(1-x)(1-y) F(x) F(y)}{2 A^{4}(x-y)^{2}}, \\
R_{3}+\zeta_{3} & =\frac{\alpha(1+x)(1-y) F(y)}{A^{2}(x-y)^{2}}, \\
R_{3}-\zeta_{3}= & \frac{\alpha(1-x)(-1-y) F(x)}{A^{2}(x-y)^{2}},
\end{aligned}
$$

$$
Y_{23}=\frac{2 \alpha^{2} F(x) F(y)}{A^{4}(x-y)^{2}} .
$$

\section{APPENDIX E: CALCULATING $\boldsymbol{\nu}$ INTEGRALS}

The purpose of this appendix is to explain how the quantity $\nu$ in Eq. (2.17) is calculated for the solutions of Sec. IV. Consider first the solution of Sec. IV E. In terms of the complex coordinates $w$, the functions $U_{i}$ take the form

$$
\begin{aligned}
& U_{1}=\log \left|\operatorname{Re}\left[\left(a_{2}+i w\right)^{1 / 2}\right]\right|-\log \left|\operatorname{Re}\left[\left(a_{1}+i w\right)^{1 / 2}\right]\right|, \\
& U_{2}=\log \left|\operatorname{Re}\left[\left(a_{3}+i w\right)^{1 / 2}\right]\right|-\log \left|\operatorname{Re}\left[\left(a_{2}+i w\right)^{1 / 2}\right]\right|, \\
& U_{3}=\log \left|\operatorname{Re}\left[\left(a_{1}+i w\right)^{1 / 2}\right]\right|+\log \left|\operatorname{Im}\left[\left(a_{3}+i w\right)^{1 / 2}\right]\right|,
\end{aligned}
$$

where the constants $a_{i}$ are defined in Eq. (4.11) and arbitrary additive constants are suppressed. To calculate $\nu$ directly, these expressions could be substituted into Eqs. (2.20) and (2.21). On the right-hand side of these equations there would be 12 terms ( 4 from each $\partial U_{i} \partial U_{j}$ ). Alternatively, one can instead deal with $\gamma$, which is determined by Eqs. (2.23) and (2.24), with only 10 terms on the right-hand side. Having obtained $\gamma, \nu$ can be immediately calculated using

$$
\nu=\gamma-\sum_{i} U_{i}
$$

Equation (2.23) takes the form

$$
\begin{aligned}
\partial \gamma= & \frac{1}{4 w}+F_{+}\left(w, \bar{w} ; a_{1}\right)+F_{+}\left(w, \bar{w} ; a_{2}\right)+F_{+}\left(w, \bar{w} ; a_{3}\right) \\
& +F_{-}\left(w, \bar{w} ; a_{3}\right)-2 G_{++}\left(w, \bar{w} ; a_{2}, a_{3}\right) \\
& +2 G_{-+}\left(w, \bar{w} ; a_{1}, a_{3}\right)+G_{++}\left(w, \bar{w} ; a_{1}, a_{3}\right) \\
& -G_{++}\left(w, \bar{w} ; a_{1}, a_{2}\right)-G_{-+}\left(w, \bar{w} ; a_{2}, a_{3}\right)
\end{aligned}
$$

where the functions $F$ and $G$ are defined by

$$
\begin{gathered}
F_{ \pm}(w, \bar{w} ; c)=-\frac{w+\bar{w}}{4(c+i w)\left[(c+i w)^{1 / 2} \pm(c-i \bar{w})^{1 / 2}\right]^{2}}, \\
G_{ \pm \pm}(w, \bar{w} ; c, d)=-\frac{w+\bar{w}}{4(c+i w)^{1 / 2}(d+i w)^{1 / 2}\left[(c+i w)^{1 / 2} \pm^{\prime}(c-i \bar{w})^{1 / 2}\right]\left[(d+i w)^{1 / 2} \pm(d-i \bar{w})^{1 / 2}\right]},
\end{gathered}
$$


where $c$ and $d$ are real constants with $c>d$. In order to integrate Eq. (E5), it is necessary to integrate $F$ and $G$. This can be done by a change of variable to $Z=(c+i w)^{1 / 2}$, which yields

$$
\begin{aligned}
\int d w F_{ \pm}(w, \bar{w} ; c)= & -\frac{1}{4} \log (c+i w)+\log \left[(c+i w)^{1 / 2}\right. \\
& \left. \pm(c-i \bar{w})^{1 / 2}\right]+\cdots,
\end{aligned}
$$

where the ellipsis denotes an arbitrary function of $\bar{w}$. One similarly obtains

$$
\begin{aligned}
& \int d w G_{++}(w, \bar{w} ; c, d) \\
&= \log \operatorname{Re}\left[(c+i w)^{1 / 2}+(d+i w)^{1 / 2}\right]-\frac{1}{2} \log \left[(c+i w)^{1 / 2}\right. \\
&+\left.(d+i w)^{1 / 2}\right]+\cdots \\
& \int d w G_{--}(w, \bar{w} ; c, d)(\mathrm{E} 9) \\
&= \log \operatorname{Im}\left[(c+i w)^{1 / 2}+(d+i w)^{1 / 2}\right]-\frac{1}{2} \log \left[(c+i w)^{1 / 2}\right. \\
&\left.+(d+i w)^{1 / 2}\right]+\cdots,(\mathrm{E} 10) \\
& \int \quad d w G_{+-}(w, \bar{w} ; c, d) \\
& \quad=\log \operatorname{Re}\left[(d+i w)^{1 / 2}\right]-\log \operatorname{Re}\left[(c+i w)^{1 / 2}\right. \\
&\left.\quad+(d+i w)^{1 / 2}\right]+\frac{1}{2} \log \left[(c+i w)^{1 / 2}\right. \\
&\left.\quad+(d+i w)^{1 / 2}\right]+\cdots,
\end{aligned}
$$

$$
\begin{aligned}
& \int d w G_{-+}(w, \bar{w} ; c, d) \\
& =\log \operatorname{Im}\left[(d+i w)^{1 / 2}\right]-\log \operatorname{Im}\left[(c+i w)^{1 / 2}\right. \\
& \left.+(d+i w)^{1 / 2}\right]+\frac{1}{2} \log \left[(c+i w)^{1 / 2}\right. \\
& \left.+(d+i w)^{1 / 2}\right]+\cdots .
\end{aligned}
$$

These results yield $\gamma$, and hence $\nu$, up to an arbitrary function of $\bar{w}$. This function can be determined up to a real constant of integration by demanding that $\nu$ be real. Finally, the following expressions can be used to express $\nu$ in terms of $R_{i}, \zeta_{i}$, and $Y_{i j}$ :

$$
R_{i}=\left|a_{i}+i w\right|
$$

$$
\begin{aligned}
R_{i}-\zeta_{i}= & \left|\operatorname{Re}\left[\left(a_{i}+i w\right)^{1 / 2}\right]\right|, \\
R_{i}+\zeta_{i}= & \left|\operatorname{Im}\left[\left(a_{i}+i w\right)^{1 / 2}\right]\right|, \\
Y_{i j}= & 2 \log \left|\operatorname{Re}\left[\left(a_{i}+i w\right)^{1 / 2}\right]\right|+2 \log \left|\operatorname{Re}\left[\left(a_{j}+i w\right)^{1 / 2}\right]\right| \\
& +4 \log \left|\left(a_{i}+i w\right)^{1 / 2}+\left(a_{j}+i w\right)^{1 / 2}\right| \\
& -4 \log \left|\operatorname{Re}\left[\left(a_{i}+i w\right)^{1 / 2}+\left(a_{j}+i w\right)^{1 / 2}\right]\right|,
\end{aligned}
$$

where additive constants have again been suppressed. The following identity is useful in rearranging $\nu$ so that it can be written in terms of the above expressions:

$$
\begin{aligned}
\log \mid \operatorname{Im} & {\left[(c+i w)^{1 / 2}+(d+i w)^{1 / 2}\right]|-\log | \operatorname{Im}\left[(d+i w)^{1 / 2}\right] \mid } \\
= & \log \left|\operatorname{Re}\left[(c+i w)^{1 / 2}+(d+i w)^{1 / 2}\right]\right| \\
& -\log \left|\operatorname{Re}\left[(c+i w)^{1 / 2}\right]\right| .
\end{aligned}
$$

[1] D. Kramer et al, Exact Solutions of Einstein's Field Equations (Cambridge University Press, Cambridge, England, 1980).

[2] R. M. Wald, General Relativity (University of Chicago Press, Chicago, 1984).

[3] H. Weyl, Ann. Phys. (Leipzig) 54, 117 (1917).

[4] S. W. Hawking and G. F. R. Ellis, The Large Scale Structure of Space-Time (Cambridge University Press, Cambridge, England, 1973).

[5] D. Gannon, J. Math. Phys. 16, 2364 (1975); Gen. Relativ. Gravit. 7, 291 (1976); J. L. Friedman, K. Schleich, and D. M. Witt, Phys. Rev. Lett. 71, 1486 (1993); 75, 1872(E) (1995); G. J. Galloway, Commun. Math. Phys. 151, 53 (1993); P. T. Chrusciel and R. M. Wald, Class. Quantum Grav. 11, L147 (1994); T. Jacobson and S. Venkataramani, ibid. 12, 1055 (1995); S. F. Browdy and G. J. Galloway, J. Math. Phys. 36, 4952 (1995); G. J. Galloway, K. Schleich, D. M. Witt, and E. Woolgar, Phys. Rev. D 60, 104039 (1999).

[6] M. Cai and G. J. Galloway, Class. Quantum Grav. 18, 2707 (2001).
[7] R. Emparan, Nucl. Phys. B610, 169 (2001).

[8] H. Leutwyler, Arch. Sci. B13, 549 (1960); P. Dobiasch and D. Maison, Gen. Relativ. Gravit. 14, 231 (1982); A. Chodos and S. Detweiler, ibid. 14, 879 (1982); D. Pollard, J. Phys. A 16, 565 (1983); G. W. Gibbons, Nucl. Phys. B207, 337 (1982); G. W. Gibbons and D. L. Wiltshire, Ann. Phys. (N.Y.) 167, 201 (1986); 176, 393(E) (1986); M. Cvetic and D. Youm, Phys. Rev. D 52, 2144 (1995); Phys. Rev. Lett. 75, 4165 (1995); D. Rasheed, Nucl. Phys. B454, 379 (1995).

[9] D. J. Gross and M. J. Perry, Nucl. Phys. B226, 29 (1983); R. D. Sorkin, Phys. Rev. Lett. 51, 87 (1983).

[10] W. Kinnersley and M. Walker, Phys. Rev. D 2, 1359 (1970).

[11] F. J. Ernst, J. Math. Phys. 17, 515 (1976).

[12] F. Dowker, J. P. Gauntlett, D. A. Kastor, and J. Traschen, Phys. Rev. D 49, 2909 (1994).

[13] G. W. Gibbons and D. L. Wiltshire, Nucl. Phys. B287, 717 (1987).

[14] S. C. Lee, Lett. Nuovo Cimento Soc. Ital. Fis. 44, 133 (1985); S. C. Lee, J. Math. Phys. 28, 893 (1987). 
[15] R. Becerril and T. Matos, Phys. Rev. D 46, 1540 (1992); T. Matos, J. Math. Phys. 35, 1302 (1994); A. Macias and T. Matos, Class. Quantum Grav. 13, 345 (1996).

[16] K. A. Bronnikov and V. N. Melnikov, Gravitation Cosmol. 1, 155 (1995); V. N. Melnikov, A. G. Radynov, and S. B. Fadeev, Russ. Phys. J. 38, 663 (1995).

[17] F. R. Tangherlini, Nuovo Cimento 77, 636 (1963).

[18] R. C. Myers and M. J. Perry, Ann. Phys. (N.Y.) 172, 304 (1986).

[19] E. Witten, Nucl. Phys. B195, 481 (1982).

[20] F. Dowker, J. P. Gauntlett, G. W. Gibbons, and G. T. Horowitz, Phys. Rev. D 52, 6929 (1995).

[21] F. Dowker, J. P. Gauntlett, G. W. Gibbons, and G. T. Horowitz, Phys. Rev. D 53, 7115 (1996).

[22] R. C. Myers, Phys. Rev. D 35, 455 (1987).

[23] D. J. Gross, M. J. Perry, and L. G. Yaffe, Phys. Rev. D 25, 330 (1982).

[24] A. Chamblin and R. Emparan, Phys. Rev. D 55, 754 (1997).
[25] R. Emparan and H. S. Reall, Phys. Rev. Lett. 88, 101101 (2002).

[26] R. B. Mann and S. F. Ross, Phys. Rev. D 52, 2254 (1995).

[27] W. Israel and K. A. Khan, Nuovo Cimento 33, 331 (1964).

[28] B. B. Godfrey, Gen. Relativ. Gravit. 3, 3 (1972).

[29] W. B. Bonnor, Gen. Relativ. Gravit. 15, 535 (1983).

[30] H. F. Dowker and S. N. Thambyahpillai, gr-qc/0105044.

[31] R. Gregory and R. Laflamme, Phys. Rev. Lett. 70, 2837 (1993).

[32] S. A. Hughes, C. R. Keeton, P. Walker, K. T. Walsh, S. L. Shapiro, and S. A. Teukolsky, Phys. Rev. D 49, 4004 (1994).

[33] S. L. Shapiro, S. A. Teukolsky, and J. Winicour, Phys. Rev. D 52, 6982 (1995).

[34] O. Lunin and S. D. Mathur, Nucl. Phys. B610, 49 (2001); R. Emparan, D. Mateos, and P. K. Townsend, J. High Energy Phys. 07, 011 (2001).

[35] K. P. Tod, Phys. Lett. 121B, 241 (1983). 